\title{
On Purely Radiative Space-Times ${ }^{(*)}$
}

\author{
Helmut Friedrich \\ Universität der Bundeswehr Hamburg, Fachbereich Maschinenbau, Holstenhofweg 85, D-2000 \\ Hamburg 70, Federal Republic of Germany
}

\begin{abstract}
The existence of space-times representing pure gravitational radiation which comes in from infinity and interacts with itself is discussed. They are characterized as solutions of Einstein's vacuum field equations possessing a smooth structure at past null infinity which forms the "future null cone at past timelike infinity with complete generators." The "pure radiation problem" is analysed where "free initial data" for Einstein's field equations are prescribed on the null cone at past time-like infinity. It is demonstrated how the pure radiation problem can be formulated as a local initial value problem for the symmetric hyperbolic system of reduced conformal vacuum field equations. Its solutions are uniquely determined by the free data.
\end{abstract}

\section{Introduction}

This paper is concerned with space-times representing gravitational radiation, which falls in from infinity, interacts nonlinearly with itself, and eventually escapes to infinity again. It is clear that a sufficiently complete understanding of this process and of the global structure of the resulting field is of fundamental importance for classical as well as for quantum gravity. Due to the work of Pirani [20], Sachs [21,22], Bondi et al [1], Newman and Penrose [12], Penrose [15-17], Geroch [10] and others, there exists a rigorous setting in which to study the situation described above, without having to take recourse to approximation procedures. One will expect that the field is a solution of Einstein's vacuum field equations which has a smooth structure at past null infinity. To make sure that the field is indeed built up only by incoming gravitational radiation, one will require that the null generators of past null infinity satisfy a certain completeness condition and that past timelike infinity is represented in the conformal completion by a point $i^{-}$. This point is required to be "regular" in the sense that the conformally rescaled "unphysical" space-time admits a smooth extension in which $i^{-}$is a regular point and such that the future null cone of $i^{-}$represents past null infinity for the physical

^ Work supported by a Heisenberg-fellowship of the Deutsche Forschungsgemeinschaft 
space-time. From the work referred to above it follows that fields with these properties have an unambiguous interpretation as representing pure gravitational radiation. If the field completely radiates away again in the future, one will expect that the space-time will satisfy the asymptotic conditions above, where "past" is replaced by "future" and " $i^{-}$" by " $i^{+}$." A solution of Einstein's vacuum field equations satisfying all the asymptotic conditions at past and future timelike and null infinity mentioned above will be called a "purely radiative space-time." These space-times are of interest because of their clear-cut physical interpretation. However, the question, whether sufficiently many purely radiative space-times exist may also be considered as a "guiding problem" in an investigation of the global propagation properties of Einstein's field equations. It appears to be the "simplest" such problem since no sources are involved.

Only one exact solution seems to be known at present to satisfy all the required conditions. In a suitably conformally rescaled form its line element is given by

$$
d s^{2}=2 d u^{2}+2 d u d r-\frac{1}{2} \sin ^{2} r d \omega^{2},
$$

where $d \omega^{2}$ denotes the standard line element on the 2-dimensional unit sphere and the coordinates $u$ and $r$ take values in the range

$$
0 \leqq u \leqq \pi, \quad 0 \leqq r \leqq \pi-u .
$$

The line $\{r=0\}$ corresponds to the usual coordinate singularity associated with a radial coordinate like $r$. Past null infinity is given by the null surface $\{u=0,0<r<\pi\}$, while future null infinity is given by $\{u+r=\pi, 0<u<\pi\}$. The points $\{u=0, r=0\},\{u=0, r=\pi\}$ and $\{u=\pi\}$ represent in that order the points $i^{-}, i^{0}$, which denotes spatial infinity, and $i^{+}$.

Rescaling the line element (1.1) by a conformal factor $\Omega^{-1}$, where $\Omega$ is given by

$$
\Omega=\sqrt{2} \sin u \sin (u+r)
$$

and performing in the range $0<u<\pi, 0 \leqq r<\pi-u$ the coordinate transformation

$$
t+r^{\prime}=-\operatorname{ctg}(u+r), \quad t-r^{\prime}=-\operatorname{ctg} u,
$$

one recovers the line element of Minkowski space in spherical polar coordinates

$$
\Omega^{-2} d s^{2}=d \tilde{s}^{2}=d t^{2}-d r^{2}-r^{2} d \omega^{2} .
$$

This state of affairs is not only deplorable because there are no exact solutions allowing one to study and to extract for possible generalization the characteristic features of gravitational waves, which interact with each other in a nonlinear though smooth way. It also may leave some doubt whether the very concept of a purely radiative space-time as depicted here is appropriate at all to model the process described in the beginning.

Minkowski space is also the only exact solution known to satisfy the "minimal condition" that a space-time representing pure gravitational radiation may possess a regular cone at past timelike infinity with complete generators. The question of the existence of solutions of Einstein's field equations satisfying these minimal conditions will be investigated in this paper by analysing the initial value problem where data are prescribed on the cone at past timelike infinity. 
The basis for this investigation are the results on the "conformal structure" of Einstein's field equations given in [5] and, more recently, on their hyperbolicity, discussed in [7]. In [5] it was shown that on the vacuum space-time Einstein's field equations can be replaced by the "regular conformal vacuum field equations" which govern the propagation of the conformally rescaled fields. The regular conformal vacuum field equations constitute a slight generalization of Einstein's equations in that they make sense also at points where the conformal factor vanishes, i.e. where Einstein's equations are not even defined. In [7] it has been shown that the propagational part of the regular conformal vacuum field equations implies a symmetric hyperbolic system of evolution equations for any choice of coordinates, frame field and Ricci scalar. Together these properties of the field equations may be considered as the structural basis for the possibility of imposing conditions on the asymptotic behaviour of the fields near past (or future) null infinity in terms of requirements on the global conformal structure.

To obtain a precise formulation of the initial value problem to be investigated, a more detailed description of the situation depicted in the beginning will be given now.

Definition (1.1). A pair $(M, g)$ is called a "Lorentz-space with a cone-like past boundary" if it can be obtained in the following way. There exists a strongly causal, oriented, and time oriented Lorentz space $\left(M^{*}, g^{*}\right)$ and a point $i^{-} \in M^{*}$ with the property that the causal future $\mathrm{J}^{+}\left(i^{-}, M^{*}\right)$ of $i^{-}$; i.e. the set consisting of $i^{-}$and all points of $M^{*}$, which can be joined to $i^{-}$by a future directed non-spacelike curve in $M^{*}$, is such that $J^{+}\left(i^{-}, M^{*}\right)$ is closed in $M^{*}$, and if $I_{1}$ - denotes the boundary $J^{+}\left(i^{-}, M^{*}\right) \cap\left(M^{*} \backslash I^{+}\left(i^{-}, M^{*}\right)\right)$ of this set, then $I=I_{1^{-}} \backslash\left\{i^{-}\right\}$is a smooth null hypersurface of $M^{*}$. Now $M$ is the set $J^{+}\left(i^{-}, M^{*}\right)$ together with all the differential structures which this set inherites from $M^{*}$, and $g$ is the pull-back of $g^{*}$ to $M$.

In particular all structures like "tangent space," "metric" etc. will retain at $i^{-} \in M$ the meaning they had on $M^{*}$. The set $I_{i}-\subset M$ will be called the (future) null cone of $i^{-}$.

Definition (1.2). A strongly causal, oriented, and time oriented Lorentz-space $(\hat{M}, \hat{g})$ is called a "solution of Einstein's vacuum field equations with complete null cone at past timelike infinity $i^{-}$," if there exists a Lorentz-space $(M, g)$ with cone-like past boundary $I_{i}$ - and a function $\Omega$ on $M$, the "conformal factor," such that

(i) $\Omega>0$ on $M \backslash I_{i^{-}} ; \Omega \equiv 0$ on $I_{i^{-}}, d \Omega \neq 0$ on $I_{i^{-}} \backslash\left\{i^{-}\right\}$, $d \Omega=0$ at $i^{-}$, but the Hessian of $\Omega$ at $i^{-}$is nondegenerate. $\}$

(ii) By a certain diffeomorphism the manifolds $\hat{M}$ and $M \backslash I_{i^{-}}$can be identified such that after the identification one has

$$
g=\Omega^{2} \hat{g} \text { on } M \backslash I_{i^{-}} .
$$

(iii) The metric $\hat{g}$ is a solution of Einstein's vacuum field equations

$$
\operatorname{Ric}(\hat{g})=0 \text { on } M \backslash I_{i}{ }^{-} \text {. }
$$

(iv) The null hypersurface $I=I_{i^{-}} \backslash\left\{i^{-}\right\}$satisfies the following completeness conditions: If the metric $g$ is rescaled by a positive conformal factor $\theta$, which 
is defined near $I$ and chosen such that the convergence of the null geodesics on $I$ with respect to the metric $\theta^{2} g$ vanishes on $I$, then the null geodesics are complete.

It is easily verified that all these conditions are satisfied by the example (1.1), (1.2), (1.3). The meaning of conditions (1.4), (1.5) has been explained in [10,15-17]. For a discussion of the significance of the completeness condition see [11]. From (1.4) follows

$$
\nabla_{\mu} \nabla_{v} \Omega=C g_{\mu v} \quad \text { at } i^{-} \text {with } C>0 .
$$

Above it is assumed, as will be done in the following, that all manifolds, functions etc. are of class $C^{\infty}$.

One can now pose the "pure radiation problem": Suppose certain data are prescribed on a cone like the one given by $\{u=0,0 \leqq<\pi\}$ of example (1.1), (1.2). Does there exist a unique solutions of Einstein's vacuum field equations with complete null cone at past timelike infinity $I_{i^{-}}$, which implies on $I_{i}$ - fields, which may in an appropriate way be identified with the given data?

There are two main difficulties which have to be overcome to obtain a satisfactory answer to the pure radiation problem. One has to find out whether it is possible to construct for appropriate, though still rather general data solutions of Einstein's vacuum field equations, which extend arbitrarily far into the past and fall-off in past null and timelike directions in such a way as to allow a smooth structure in the infinite past and to induce the given data there. Furthermore, data are given on a surface which is not smooth at the vertex. Though at first sight the last point appears to be of minor importance, it turns out that it is the nonsmoothness of the initial surface which creates all the technical problems and prevents one from deriving an existence result in the case of low differentiability by more or less straightforward application of standard techniques.

Initial value problems for Einstein's field equations, where data are prescribed on a cone which is to represent the future light cone of a regular point of space-time, have been analysed in $[2,4,14]$, emphasizing the question which data may be prescribed in that case. Detailed existence results referring to this situation have not been worked out yet. There are available techniques to show the existence of solutions for linear systems of wave-equations if data are given on a cone [3]; however there do not seem to exist in the literature similar results which will readily apply to the present quasilinear case.

It will be shown in this paper (Propositions (9.2), (9.3)) that the pure radiation problem can be formulated as an initial value problem for a symmetric hyperbolic system and that, in a given choice of gauge, a solution to this problem is uniquely determined by the free data (the "radiation field"). As a byproduct it follows that the data determine a unique formal expansion of the fields. The basic assumption which enters into this discussion is that not only $\Omega^{2} \hat{g}_{\mu \nu}$ but also $\Omega^{-1} \hat{C}^{\mu}{ }_{\lambda \rho \delta}$ extends to a smooth tensor field on the non-physical space-time, where $\hat{C}_{\lambda \rho \delta}^{\mu}$ denotes the Weyl tensor.

The analysis requires the use of two different types of gauge conditions. To formulate a regular initial value problem one needs smooth coordinates and frame 
fields defined in a neighbourhood of the initial cone. This will be discussed in Chap. 9. However, the analysis near the vertex $i^{-}$of the interior equations which are implied on the initial cone by the field equations requires the use of coordinates and frame fields which will become singular on a timelike geodesic which passes through the vertex $i^{-}$. The initial surface will be a level surface of a null coordinate and three of the vector fields which constitute the frame field will be tangent to that surface.

Much of the following work is needed to develop methods which make it possible to handle the field equations in this singular gauge. The various fields which will be determined from the data will appear singular because of the singular coordinates and the singular frame. The knowledge, whether the resultant solution space-time will be smoothly extensible through the singular line has to be encoded into the form of the initial data. This makes it necessary to study regularity conditions on the cone near the vertex.

The natural place for such a discussion turns out to be the bundle $S(M)$ of spin frames. Therefore Chaps. 1-4 are concerned with the formulation of the regular conformal vacuum field equations as equations of forms on $S(M)$, with the construction of a certain 5-dimensional submanifold $\tilde{M}$ of $S(M)$, which arises naturally in the present context, and with the choice of coordinates and frame vector fields on $\tilde{M}$. In Chap. 5 will be discussed smoothness conditions on the cone for the various fields. Here and in Chaps. 6 and 8, which deal with the field equations on $\tilde{M}$, it is seen that the setting provided by the manifold $\tilde{M}$ allows us in a neat way to exploit the group theoretical content of the fields and the field equations.

One reason for the present formulation of the problem was the wish to say more about the existence of solutions of the pure radiation problem in the case of analytic data. If one wants to show the convergence of the formal expansion of the fields referred to in Proposition (8.1), the field equations must be written in a form which gives a sufficiently explicit description of the iteration procedure by which the expansion coefficients are calculated. This form is obtained in Chaps. 6 and 8. In a subsequent paper [8] conditions will be stated under which the formal expansion discussed in Proposition (8.1) does in fact provide an analytic solution of the conformal vacuum field equations.

The reduction of the pure radiation problem to the initial problem formulated in Chap. 9 leaves one with the task of proving the existence of solutions if data of low differentiability are given. But in spite of the remaining technical problems already the results presented in this paper show that the concept of a solution of Einstein's vacuum field equations with complete null cone at past timelike infinity is in perfect harmony with the conformal structure of Einsteins field equations.

In an investigation of the global behaviour of the "local solutions" near $I_{i}$ obtained from the pure radiation problem it may turn out that even with certain restrictions on the data most of the solutions will evolve into something which looks more exotic than a purely radiative space-time. Black holes may form or the solution may simply fail to allow a smooth structure at future null infinity. However the final answer will turn out, the attempt to arrive at a well-founded statement on this will 
deepen our understanding of the non-linear interaction of gravitational waves and their propagation over large regions of space-time.

\section{The Conformal Vacuum Field Equations}

In the following the conformal vacuum field equations as discussed in [7] will be written as system of equations of forms on the bundle of spinframes $S(M)$ over a four dimensional manifold $M$ with conelike past boundary $I_{i}{ }^{-}$ as described in Definition (1.2). All considerations will refer to a fixed choice of the unphysical metric $g$ on $M(\operatorname{sign}(+,-,-,-))$. The notation and the formalism will follow closely that of $[5,7]$.

An element of the bundle $S(M)$ is a normalized spinframe $\delta=\left(\delta_{a}\right)_{a=0,1}$ at some point $x$ in $M$, such that

$$
\varepsilon\left(\delta_{a}, \delta_{b}\right)=\varepsilon_{a b},
$$

where $\varepsilon$ is the antisymmetric form on the spinor bundle. The antisymmetric Levi-Civita symbols $\varepsilon_{a b}$, $\varepsilon^{a b}$ satisfying $\varepsilon_{01}=\varepsilon^{01}=1$ are used for lowering and raising indices. The projection $\pi: S(M) \rightarrow M$ maps the spinframe $\delta$ onto its base point $x$ in $M$. The group $\operatorname{SL}(2, \mathbb{C})$ of all complex $2 \times 2$ matrices $t=\left(t_{b}^{a}\right)$ satisfying $\operatorname{det}\left(t^{a}{ }_{b}\right)=1$ acts transitively on the fibres $\pi^{-1}(x)$ of $S(M)$ by

$$
\delta=\left(\delta_{a}\right) \rightarrow\left(\delta_{b} t_{a}^{b}\right)=\delta \cdot t \quad \forall \delta \in S(M), \quad t \in \mathrm{SL}(2, \mathbb{C}),
$$

such that $\pi(\delta \cdot t)=\pi(\delta)$. Let $\sigma_{a a^{\prime}}^{j}, \sigma_{k}^{b b^{\prime}}$ be the constant matrices defined by the map

$$
\mathbb{R}^{4} \ni\left(x^{k}\right) \rightarrow x^{k} \sigma_{k}{ }^{a a^{\prime}}=\frac{1}{\sqrt{2}}\left(\begin{array}{cc}
x^{0}+x^{3} & x^{1}+i x^{2} \\
x^{1}-i x^{2} & x^{0}-x^{3}
\end{array}\right)=x^{a a^{\prime} \in M_{2 \times 2},}
$$

which identifies $\mathbb{R}^{4}$ with the set of hermitian $2 \times 2$ matrices, and the conditions

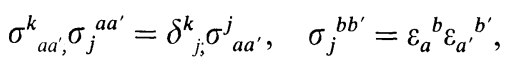

The twofold covering homomorphism of the group SL $(2, \mathbb{C})$ onto the group $L_{+}^{\uparrow}$ of all proper Lorentz transformations (component containing the unit element) is then realized by

$$
\operatorname{SL}(2, \mathbb{C}) \ni t^{a}{ }_{b} \stackrel{\psi}{\rightarrow} \sigma_{a a^{\prime}}^{i} t^{a}{ }_{b} \bar{t}_{{ }^{\prime}}{ }^{\prime}{ }{ }^{b b^{\prime}}=t^{i}{ }_{k} \in L^{\dagger}+.
$$

The spinors $\delta_{a} \delta_{a^{\prime}}$ associated with a spinframe $\delta$ correspond to a null tetrad $\left(c_{a a^{\prime}}\right)_{a, a^{\prime}=0,1}$ satisfying

$$
g\left(c_{a a^{\prime}}, c_{b b^{\prime}}\right)=\varepsilon_{a b} \varepsilon_{a^{\prime} b^{\prime}}, \quad c_{a a^{\prime}}=\bar{c}_{a a^{\prime}},
$$

which in turn determines an orthonormal frame $c_{k}=c_{a a^{\prime}} \sigma_{k}^{a a^{\prime}}, k=0,1,2,3$. The map

$$
\left(\delta_{a}\right) \stackrel{\Psi}{\rightarrow}\left(c_{k}\right)
$$

is a twofold covering bundle morphism of the bundle of normalized spin frames onto 
the bundle of oriented and time oriented $g$-orthonormal frames, such that

$$
\Psi(\delta \cdot t)=\Psi(\delta) \cdot \psi(t) \quad \forall \delta \in S(M), t \in \operatorname{SL}(2, \mathbb{C}) .
$$

The unknowns in the conformal vacuum field equations will be expressed in terms of some basic 1-forms on $S(M)$ and in terms of "spinor valued functions" on $S(M)$.

The basic 1-forms are:

- the solder form $\sigma^{a a^{\prime}}$, which takes value in the set of hermitian $2 \times 2$ matrices, i.e. $\sigma^{a a^{\prime}}=\bar{\sigma}^{a a^{\prime}}$. It is obtained from the solder form $\sigma^{i}$ on the bundle of orthonormal frames as a pull-back,

$$
\sigma^{a a^{\prime}}=\left({ }^{t} \Psi \sigma^{k}\right){\sigma_{k}}^{a a^{\prime}},
$$

or can be defined, once the relation between $\delta_{a} \bar{\delta}_{a^{\prime}}$ and $c_{a a^{\prime}}$ is given, by

$$
\left\langle\sigma^{a a^{\prime}}, h\right\rangle c_{a a^{\prime}}=T(\pi) h,
$$

where $h$ is a tangent vector of $S(M)$ at the point $\delta$. This formula shows that $\sigma^{a a^{\prime}}$ supplies a basis of horizontal 1-form, i.e. of those forms which vanish on vectors tangent to the fibres of $S(M)$;

- the connection form $\omega^{a}{ }_{b}$ which takes values in the Lie algebra $\operatorname{sl}(2, \mathbb{C})$ of all complex traceless $2 \times 2$ matrices. It is obtained from the connection form $\omega^{i}{ }_{k}$ on the bundle of orthonormal frames by a pull-back operation

$$
\omega_{b}^{a}=\frac{1}{2}\left({ }^{t} \Psi \omega_{k}^{i}\right) \sigma_{i}^{a d^{\prime}} \sigma_{b d^{\prime}}^{k}
$$

Each spinor field on $M$ gives rise to a spinor valued function on $S(M)$, which at a point $\delta \in S(M)$ is given by the coefficients of the spinorfield with respect to the spin frame $\left(\delta_{a}\right)$. If these are given by $\phi_{a b c \ldots a^{\prime} b^{\prime} c^{\prime} \ldots}(\delta)$, then under the action of $\operatorname{SL}(2, \mathbb{C})$ on the fibres on $S(M)$ they transform according to

$$
\phi_{a b \ldots a^{\prime} b^{\prime} \ldots}(\delta \cdot t)=\phi_{c d \ldots c^{\prime} d^{\prime} \ldots}(\delta) t^{c}{ }_{a} t^{d}{ }_{b} \ldots \bar{t}^{c^{\prime}}{ }_{a^{\prime}} \bar{t}^{d^{\prime}}{ }_{b^{\prime} \ldots} .
$$

Forms on $S(M)$ which under fibre transformations transform in this way (if all indices are lowered) will be called invariant. Scalar functions on $M$ define invariant functions on $S(M)$ which are constant on the fibres. The following functions on $S(M)$ are needed to formulate the conformal vacuum field equations.

- the conformal factor $\Omega$, the Ricci scalar $R$, respectively $\Lambda=\frac{1}{24} R$, and the function $s=\frac{1}{4} \nabla_{\mu} \nabla^{\mu} \Omega$. That the functions $\Omega$ and $s$ are constant on the fibres will follow from the field equations, the constancy of $\Lambda$ on the fibres will be assumed everywhere;

- the spinor valued functions $\Sigma_{a a^{\prime}}=\bar{\Sigma}_{a a^{\prime}}, \phi_{a b a^{\prime} b^{\prime}}=\phi_{(a b)\left(a^{\prime} b^{\prime}\right)}=\bar{\phi}_{a b a^{\prime} b^{\prime}}, \varphi_{a b c d}=\varphi_{(a b c d)}$ which represent, in that order, the differential of the function $\Omega$, the traceless part of the Ricci tensor, and the rescaled Weyl spinor $\Omega^{-1} \Psi_{a b c d}$.

The following horizontal forms will appear in the field equations beside $\sigma^{a a^{\prime}}$ :

$$
\begin{aligned}
\Sigma & =\Sigma_{a a^{\prime}} \sigma^{a a^{\prime}}, \Sigma_{a b}=\Sigma_{(a}^{h^{\prime}} \sigma_{b) h^{\prime}}, \\
\varphi_{a b} & =-\frac{1}{2} \varphi_{a b c d} \sigma^{c h^{\prime}} \wedge \sigma_{h^{\prime}} \\
\phi_{a b} & =-\frac{1}{2} \phi_{a b c^{\prime} d^{\prime}} \sigma^{h c^{\prime}} \wedge{\sigma_{h}{ }^{d^{\prime}}} \\
s_{a b} & =s \sigma_{a}^{h^{\prime}} \wedge \sigma_{b h^{\prime}} .
\end{aligned}
$$


These forms together with the solder and the connection forms are invariant, i.e. under the pull-back implied by the fibre transformation $\delta \rightarrow \delta \cdot t$ they transform according to (2.1). Let $D$ denote the covariant differential on $S(M)$. If it is applied to an invariant horizontal spinor valued form $\eta_{a b c^{\prime}}$ it gives an expression

$$
D \eta_{a b c^{\prime}}=d \eta_{a b c^{\prime}}-\omega_{a}^{f} \wedge \eta_{f b c^{\prime}}-\omega_{b}^{f} \wedge \eta_{a f c^{\prime}}-\bar{\omega}_{c^{\prime}}^{f^{\prime}} \wedge \eta_{a b f^{\prime}}
$$

The conformal vacuum field equations can now be written

$$
\begin{aligned}
& D \sigma^{a a^{\prime}}=0 \\
& D \omega_{b}^{a}=\Omega_{b}^{a} \text { with } \quad \Omega^{a}{ }_{b}=\Omega \varphi^{a}{ }_{b}+\phi^{a}{ }_{b}+\Lambda \sigma^{a h^{\prime}} \wedge \sigma_{b h^{\prime}}, \\
& D \varphi_{a b}=0 \text {, } \\
& D \phi_{a b}=-\Sigma \wedge \varphi_{a b}-d \Lambda \wedge \sigma_{a}^{h^{\prime}} \wedge \sigma_{b h^{\prime}}, \\
& D \Sigma_{a b}=-\Omega \phi_{a b}+s_{a b} \text {, } \\
& D \Omega=\Sigma \text {, } \\
& D \Sigma=0 \text {, } \\
& D s_{a b}=\Sigma \wedge \phi_{a b}-2 \Sigma_{f(a} \wedge \phi_{b)}{ }^{f} \\
& -2 \Lambda \Sigma \wedge \sigma_{a}^{h^{\prime}} \wedge \sigma_{b h^{\prime}}-\Omega d \Lambda \wedge \sigma_{a}^{h^{\prime}} \wedge \sigma_{b h^{\prime}}, \\
& \Omega s_{a b}+\Sigma_{f a} \wedge \Sigma_{b}^{f}+\Sigma_{a b} \wedge \Sigma+\Omega^{2} \Lambda \sigma_{a}^{h^{\prime}} \wedge \sigma_{b h^{\prime}}=0 .
\end{aligned}
$$

Using the star operator Eqs. (2.5), (2.6) may be written equivalently

$$
\begin{aligned}
& { }^{*} D^{*} \varphi_{a b}=0, \\
& { }^{*} D^{*} \phi_{a b}=-i^{*}\left(\Sigma \wedge \varphi_{a b}+d \Lambda \wedge \sigma_{a}{ }^{h^{\prime}} \wedge \sigma_{b h^{\prime}}\right) .
\end{aligned}
$$

If one chooses a local section $x \rightarrow\left(\delta_{a}(x)\right)$ of $S(M)$ over $M$ and expresses the pull-backs of Eqs. (2.3), (2.4), (2.7)-(2.13) by this section in terms of the tetrad field $c_{a a^{\prime}}(x)$ associated with the spinframe field $\delta_{a}(x)$, then the conformal vacuum field equations take the form in which they are presented in [7]. The scalar $\Lambda$ is considered here as an arbitrarily given smooth function.

The Eqs. (2.3), (2.4) are just the structure equations with the torsion form required to vanish and the curvature form decomposed into its irreducible parts. Equation (2.5) is the vacuum Bianchi identity expressed in terms of the rescaled Weyl spinor and the structures derived from the non-physical metric $g$. Equation (2.6) is obtained from the Bianchi identity for the curvature form $\Omega^{a}{ }_{b}$ by taking into account the decomposition of the curvature form and Eq. (2.5). Equation (2.7) is the traceless part of Einstein's vacuum field equations $\operatorname{Ric}\left(\Omega^{-2} g\right)=0$. Equation (2.8) is the definition of $\Sigma$ and (2.9) a trivial consequence. Equation (2.10) is obtained by applying the covariant differential to (2.7) and using (2.3)-(2.9). Finally the trace of the equation $\operatorname{Ric}\left(\Omega^{-2} g\right)=0$ is given by (2.11).

If one defines

$$
\tau_{a b}=\Omega s_{a b}+\Sigma_{f a} \wedge \Sigma_{b}^{f}+\Sigma_{a b} \wedge \Sigma+\Omega^{2} \Lambda \sigma_{a}^{h^{\prime}} \wedge \sigma_{b h^{\prime}}
$$


and assumes that only Eqs. (2.3)-(2.10) are satisfied, then from these equations one deduces

$$
D \tau_{a b}=0
$$

Because $\tau_{a b}$ is of the form $\tau_{a b}=\tau \sigma_{a}^{b^{\prime}} \wedge \sigma_{b h^{\prime}}$ with

$$
\tau=\bar{\tau}=\Omega S-\frac{1}{2} \Sigma_{a a^{\prime}} \Sigma^{a a^{\prime}}+\Omega^{2} \Lambda,
$$

Eq. (2.15) can in view of (2.3) be written

$$
d \tau \wedge \sigma_{a}^{h^{\prime}} \wedge \sigma_{b h^{\prime}}=0
$$

Since the $\sigma_{a}^{h^{\prime}} \wedge \sigma_{b h^{\prime}}$ together with their complex conjugates form a basis of the horizontal 2-form, one concludes $d \tau=0$, i.e.

$$
\Omega s-\frac{1}{2} \Sigma_{a a^{\prime}} \Sigma^{a a^{\prime}}+\Omega^{2} \Lambda=: \hat{\Lambda}=\mathrm{const}
$$

on any connected component of $S(M)$. For this constant one has $\hat{\Lambda}=\frac{1}{24} \hat{R}$. On the subset of $M$ where $\Omega \neq 0 \hat{R}$ is the Ricci scalar of the space-time given by $\Omega^{-2} g_{\mu \nu}$. This metric is required to be a solution of Einstein's vacuum field equations (with vanishing cosmological constant). If the data for the initial value problem for Eqs. (2.3)-(2.10) are given such that they satisfy the condition (1.4), then $\hat{\Lambda}$ vanishes at $i^{-}$and Eq. (2.11) will be satisfied as a consequence of the other Eqs. (2.3)-(2.10).

\section{The Manifold $\tilde{M}$}

For given $x=\left(x^{k}\right) \in \mathbb{R}^{4}$ and $u=\left(u^{a}{ }_{b}\right) \in \operatorname{sl}(2, \mathbb{C})$ the horizontal vector field $H_{x}$ and the vertical vector field $Z_{u}$ are defined on $S(M)$ by the conditions

$$
\begin{array}{cc}
\left\langle\sigma^{a a^{\prime}}(\delta), H_{x}(\delta)\right\rangle=x^{a a^{\prime}} ; & \left\langle\omega^{a}{ }_{b}(\delta), H_{x}(\delta)\right\rangle=0 ; \\
\left\langle\sigma^{a a^{\prime}}(\delta), Z_{u}(\delta)\right\rangle=0 ; & \left\langle\omega^{a}{ }_{b}(\delta), Z_{u}(\delta)\right\rangle=u^{a}{ }_{b} ; \\
\forall \delta \in S(M) . &
\end{array}
$$

At $\delta \in S(M)$ the tangent vector $H_{x}(\delta)$ is the unique horizontal vector with projection $T(\pi) H_{x}(\delta)=x^{a a^{\prime}} c_{a a^{\prime}}(\delta)$. In the following $H_{a a^{\prime}}$ will denote that horizontal vector field which satisfies $\left\langle\sigma^{b b^{\prime}}, H_{a a^{\prime}}\right\rangle=\varepsilon_{a}{ }^{b} \varepsilon_{a^{\prime}}{ }^{\prime}$, or equivalently $T(\pi) H_{a a^{\prime}}(\delta)=$ $c_{a a^{\prime}}(\delta)$. For $\delta \in S(M)$ consider the geodesic through $\pi(\delta)$ with tangent vector $T(\pi) H_{x}(\delta)$. Then the integral curve of $H_{x}$ through $\delta$ is given by the spinframe field obtained by parallel transport of the spinframe $\delta$ along that geodesic. The integral curves of the vector field $Z_{u}$ are the orbits of the 1-parameter subgroup of $\operatorname{SL}(2, \mathbb{C})$ generated by $u$.

The horizontal vector fields may be used to give a simple description of the construction of the manifold $\tilde{M}$ and of a coordinate system on it. Let $\left(\delta_{a}\right)$ be an arbitrarily chosen normalized spin frame at $i^{-}$and $\delta^{a a^{\prime}}$ be such that

$$
\delta^{a a^{\prime}} \delta_{a} \delta_{a^{\prime}}=\delta_{0} \delta_{0^{\prime}}+\delta_{1} \bar{\delta}_{1^{\prime}}
$$

Denote by $\mathrm{SU}(2)$ the subgroup of all $t=\left(t_{b}^{a}\right) \in \mathrm{SL}(2, \mathbb{C})$ satisfying

$$
\delta_{a a^{\prime}} t^{a}{ }_{b} t_{b^{\prime}}^{a^{\prime}}=\delta_{b b^{\prime}}
$$


Furthermore denote by $\delta(t)$ the spinframe $\left(\delta_{a} t^{a}{ }_{b}\right)$ with $t \in \mathrm{SU}(2)$ and let $u \rightarrow \delta(u, t)$ the integral curve of the vector field $H_{00^{\prime}}+H_{11^{\prime}}$, through the point $\delta(t)$, where the parameter $u$ of this curve is chosen such that $\delta(0, t)=\delta(t)$. Finally let $r \rightarrow \delta(u, r, t)$ denote the integral curve of the vector field $H_{00}$, through $\delta(u, t)$, where the parameter $r$ satisfies $\delta(u, 0, t)=\delta(u, t)$.

The points $\delta(u, r, t)$ of $S(M)$ with $u \geqq 0, r \geqq 0$ sweep out a smooth five-dimensional submanifold $\tilde{M}$ of $S(M)$, which has a boundary and an edge and which is diffeomorphic to $\mathrm{SU}(2) \times \mathbb{R}_{0}^{+} \times \mathbb{R}_{0}^{+}$. It is smoothly parametrized by $u, r$, and $t \in \mathrm{SU}(2)$ and any coordinate system $z^{A}=z^{A}(t)$ on SU(2) implies a coordinate system on $\tilde{M}$. In such a coordinate system the vector fields $\partial_{u}, \partial_{r}$ are defined and their definition is independent of the choice of coordinates $z^{A}$ on $\mathrm{SU}(2)$.

One has

$$
\partial_{r}=H_{00^{\prime}} \text { on } \tilde{M}, \partial_{u}=H_{00^{\prime}}+H_{11^{\prime}} \text { on }\{r=0\},\left[\partial_{r}, \partial_{u}\right]=0 \text { on } \tilde{M} \text {, }
$$

which may also be taken as the definition of the vector fields $\partial_{u}, \partial_{r}$. The boundary of $\tilde{M}$ is formed by the sets $\{u=0\}$ and $\{r=0\}$, which are both diffeomorphic to $\mathrm{SU}(2) \times \mathbb{R}_{0}^{+}$and intersect at the edge $\{u=0, r=0\} \cong \mathrm{SU}(2)$. Here, of course, only the manifold structure of SU(2) is referred to, the group structure has no meaning on the manifold $\tilde{M}$. However some relics of the action of SU(2) on $S(M)$ are transferred to $\tilde{M}$. The sets $\{r=0, u=$ const $\}$ are contained completely in the fibres of $S(M)$ and are invariant under the action of SU(2). The action implies various structures on the sets $\{r=0, u=$ const $\}$, which may be dragged along with the vector field $H_{00^{\prime}}$ and thus be defined on all the 3-spheres $\{r=$ const, $u=$ const $\}$. Let $U(1)$ denote the subgroup of SU(2) given by the elements of the form

$$
s^{a}{ }_{b}=s^{a}{ }_{b}(\phi)=e^{\frac{1}{2} \phi} \varepsilon_{0}{ }^{a} \varepsilon_{b}{ }^{0}+e^{-\frac{1}{2} i \phi} \varepsilon_{1}{ }^{a} \varepsilon_{b}{ }^{1} ; \phi \in \mathbb{R} .
$$

Since the vector field $H_{00^{\prime}}$ is invariant under the transformations of $S(M)$ implied by the elements of $U(1)$ (see (4.4)), the group $U(1)$ acts not only on the subsets $\{r=0, u=$ const $\}$ but everywhere on $\tilde{M}$. The restriction of the projection $\pi$ to $\tilde{M}$ defines a smooth map $\tilde{\pi}$ of $\tilde{M}$ into $M$. The image of $\tilde{\pi}$ may cover $M$ to a large extent but for the present purpose it is sufficient that $\tilde{\pi}(\tilde{M})$ contains a neighbourhood of $I_{i^{-}}$in $M$. Let $c_{a a^{\prime}}(u, r, t)$ denote the null tetrad associated with the spinframe $\delta_{a}(u, r, t)$. The vector $\delta^{a a^{\prime}} c_{a a^{\prime}}(u, 0, t)$ is independent of $t \in \mathrm{SU}(2)$ and it is the tangent vector to a time-like geodesic $\gamma=\gamma(u)$ with affine parameter $u$ such that $\gamma(0)=i^{-}$. The point set $\{\gamma\} \subset M$ of points run through by the geodesic $\gamma$ is the image under $\tilde{\pi}$ of the set $\{r=0\} \subset \tilde{M}$. For fixed $t \in \operatorname{SU}(2)$ the frame $c_{a a^{\prime}}(u, 0, t)$ is parallely transported along $\gamma$. The set of null vectors $\left\{c_{00^{\prime}}(u, 0, t) /\right.$ $t \in \mathrm{SU}(2)\} \cong S^{2}$ contains all future-directed null vectors $l$ at $\gamma(u)$ satisfying $g\left(l, \gamma^{\prime}(u)\right)=$ 1. For $s \in \mathrm{SU}(2)$ one has $c_{00^{\prime}}(u, 0, t)=c_{00^{\prime}}(u, 0, t \cdot s)=c_{a a^{\prime}}(u, 0, t) s_{0}^{a} a_{0^{\prime}}$, if and only if $s \in U(1)$. Thus the map $\pi^{\prime}$ which maps $\delta(u, 0, t)$ onto $c_{00^{\prime}}(u, 0, t)$ for all $t \in \mathrm{SU}(2)$ may be identified with the Hopf-fibration $\mathrm{SU}(2) \rightarrow \mathrm{SU}(2) / U(1) \cong S^{2}$. Of particular interest are the smooth 4-dimensional submanifolds $\widetilde{N}_{u_{0}}=\left\{u=u_{0}\right\}$ of $\tilde{M}$, which are generated by the integral curves $r \rightarrow \delta(u, r, t), t \in \mathrm{SU}(2)$, of $H_{00}$. These curves project onto the null geodesics starting with tangent vector $c_{00},(u, 0, t)$ at $\gamma(u)$, such that $r$ is an affine parameter which vanishes at $\gamma(u)$. Two such curves $r \rightarrow \delta(u, r, t)$, $r \rightarrow \delta(u, r, t \cdot s)$ project onto the same null geodesic if and only if $s \in U(1)$. For fixed 
$u$ and $t$ the frame $c_{a a^{\prime}}(u, r, t)$ is obtained by parallel transport of $c_{a a^{\prime}}(u, 0, t)$ along this geodesic such that in particular $c_{00}(u, r, t)$ coincides with its tangent vector. It ensues that $\tilde{N}_{u}$ projects onto the null cone $N_{\gamma(u)}$ generated by the future directed null geodesics emanating from $\gamma(u)$. In particular one has $\tilde{\pi}\left(\tilde{N}_{0}\right)=I_{i^{-}}=N_{\gamma(0)}$. The action of $U(1)$ on $\tilde{M}$, which is reflected in the frame $c_{a a^{\prime}}(u, r, t)$ by a rotation in the $c_{01^{\prime}}-c_{10^{\prime}}$-plane, induces a factorization of the map $\tilde{\pi}$ in the form

$$
\tilde{M} \stackrel{\pi_{1}}{\rightarrow} \tilde{M} / U(1) \stackrel{\pi_{2}}{\rightarrow} M .
$$

The maps $\pi_{1}, \pi_{2}$ are smooth and the map $\pi_{1}$ has rank equal to 4 everywhere.

The manifold $\tilde{M} / U(1)$ may be identified with the Lagrange manifold (see [9] for this notion and a general discussion of the behaviour of this Lagrange manifold near caustics in the present setting), which is swept out in the tangent bundle of $M$ by the null vectors $c_{00}(u, r, t)$, while $\pi_{2}$ is the corresponding Lagrange map. There exists neighbourhoods $U$ of $\pi_{2}^{-1}(\{\gamma\})$ and $V$ of $\{\gamma\}$, the latter containing a neighbourhood of $I_{i-}$, such that $\pi_{2}$ maps $U \backslash \pi_{2}^{-1}(\{\gamma\})$ diffeomorphically onto $V \backslash\{\gamma\}$. The surface $N_{\gamma(u)} \cap(V \backslash\{\gamma\})$ is a smooth null hypersurface such that $\delta\left(u^{\prime}, r, t\right)$ is mapped by $\tilde{\pi}$ into this surface only if $u^{\prime}=u$. Thus $u$ may be considered as a smooth function on $V \backslash\{\gamma\}$, with the above null hypersurface being a level surface of $u$. Similarly $r$ may be considered as a smooth function on $V \backslash\{\gamma\}$. Following the null geodesics which generate $N_{\gamma(u)}$ further out, one may encounter caustics and $u$ and $r$ cannot be used as coordinates on $M$ any longer. The set $\{\delta \in \tilde{M} / \operatorname{rank}(T(\tilde{\pi}))<4\}$ will be called the "caustic set" of $M$. It contains in particular the set $\{r=0\}$. The image of the caustic set under $\tilde{\pi}$ is the set of points in $M$ where the null geodesics generating the sets $N_{\gamma(u)}$ develop envelopes, the null cones on $\gamma$ being particular degenerate cases.

\section{The Construction of the Frame on $\tilde{M}$}

Taking the pull-back to $\tilde{M}$ of a smooth form on $S(M)$ yields a smooth form on $\tilde{M}$ which will be denoted by the same symbol as the original form. Thus the field equations are obtained on $\tilde{M}$ as they are represented by Eqs. (2.3)-(2.11). These will be expressed later with respect to a certain frame on $\tilde{M}$ which will be constructed now.

The action $x^{a a^{\prime}} \rightarrow t^{a}{ }_{b} \bar{t}^{a^{\prime}}{ }_{b^{\prime}} x^{b b^{\prime}}$ of $\operatorname{SL}(2, \mathbb{C})$ on $\mathbb{R}^{4}$ implies an action $x^{a a^{\prime}} \rightarrow u_{b}^{a} x^{b a^{\prime}}+\bar{u}^{a^{\prime}}{ }_{b^{\prime}} x^{a b^{\prime}}=\varphi(u) \cdot x$ on the Lie-algebra sl $(2, \mathbb{C})$ on $\mathbb{R}^{4}$. It is important to note that the map

$$
\operatorname{sl}(2, \mathbb{C}) \ni u \rightarrow \varphi(u) \in \operatorname{End}\left(\mathbb{R}^{4}\right)
$$

is $\mathbb{R}$-linear but not $\mathbb{C}$-linear. This may create difficulties if $\operatorname{sl}(2, \mathbb{C})$ is considered as the complexification of $\operatorname{su}(2)$. Hence $\operatorname{sl}(2, \mathbb{C})$ must in the following relations (4.1) be treated as a real 6-dimensional Lie-algebra with real 3-dimensional subalgebra $\mathrm{su}(2)$. In most of the following considerations only the group SU(2) and its Liealgebra will be involved so that no problems will arise.

From the structure Eqs. (2.3), (2.4), the relations (3.1), and the fact that the 
curvature form $\Omega^{a}{ }_{b}$ is horizontal, one concludes

$$
\begin{aligned}
& {\left[Z_{u}, H_{x}\right]=H_{\varphi(u) x},} \\
& {\left[Z_{u_{1}}, Z_{u_{2}}\right]=Z_{\left[u_{1}, u_{2}\right]},} \\
& \left\langle\sigma^{a a^{\prime}},\left[H_{x} \wedge H_{y}\right]\right\rangle=0, \\
& \left\langle\omega^{a}{ }_{b},\left[H_{x} \wedge H_{y}\right]\right\rangle=-\left\langle\Omega^{a}{ }_{b}, H_{x} \wedge H_{y}\right\rangle, \\
& \forall u, u_{1}, u_{2} \in \operatorname{sl}(2, \mathbb{C}), x, y \in \mathbb{R}^{4},
\end{aligned}
$$

where $\left[u_{1}, u_{2}\right]$ denotes the commutator of $u_{1}, u_{2}$ in $\operatorname{sl}(2, \mathbb{C})$. If $\mathscr{L}_{x}$ is the Lie-derivative in the direction of $H_{x}$, one obtains from (4.1), (3.1),

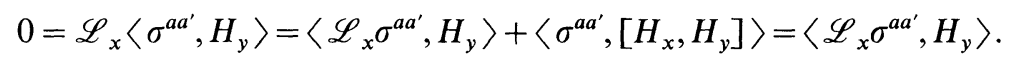

From similar calculations where $H_{y}$ is replaced by $Z_{u}$ and then $\sigma^{a a^{\prime}}$ by $\omega^{a}{ }_{b}$ one obtains on $S(M)$,

$$
\begin{aligned}
& \left\langle\mathscr{L}_{x} \sigma^{a a^{\prime}}, \cdot\right\rangle=\left\langle\omega^{a}{ }_{b}, \cdot\right\rangle x^{b a^{\prime}}+\left\langle\bar{\omega}^{a^{\prime}}{ }_{b^{\prime}}, \cdot\right\rangle x^{a b^{\prime}}, \\
& \left\langle\mathscr{L}_{x} \omega^{a}{ }_{b}, \cdot\right\rangle=\left\langle\Omega^{a}{ }_{b}, H_{x} \wedge \cdot\right\rangle .
\end{aligned}
$$

No particular field equations have been used in the derivation of these relations, it is only assumed that the torsion form vanishes.

A basis of the Lie algebra of $\mathrm{SU}(2)$ is given by

$$
h=\frac{1}{2}\left(\begin{array}{rr}
i & 0 \\
0 & -i
\end{array}\right), \quad u_{1}=\frac{1}{2}\left(\begin{array}{ll}
0 & i \\
i & 0
\end{array}\right), \quad u_{2}=\frac{1}{2}\left(\begin{array}{rr}
0 & -1 \\
1 & 0
\end{array}\right) \text {. }
$$

Since $h$ is a generator of the group $U(1)$, defined by (3.5), which acts on $\tilde{M}$, the complex vector field $S=-2 i Z_{h}$ on $S(M)$ is "tangent" to $\tilde{M}$. The kernel of the map $T(\tilde{\pi})$ contains the space $\mathbb{R} \cdot Z_{h}$ and coincides with that space for $r>0, r$ not too large. From (3.1), (4.1) one finds

$$
\begin{aligned}
& \left\langle\sigma^{a a^{\prime}}, S\right\rangle=0, \quad\left\langle\omega^{a}{ }_{b}, S\right\rangle=k^{a}{ }_{b}:=\varepsilon_{0}{ }^{a} \varepsilon_{b}{ }^{0}-\varepsilon_{1}{ }^{a} \varepsilon_{b}{ }^{1},
\end{aligned}
$$

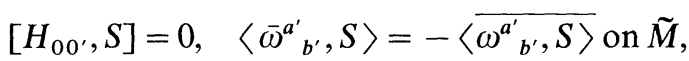

furthermore

$$
\left[H_{00^{\prime}}+H_{11^{\prime}}, S\right]=0 \text { on }\{r=0\} \text {, whence }\left[\partial_{u}, S\right]=0 \text { on } \tilde{M} \text {. }
$$

The vector fields $Z_{u_{1}}, Z_{u_{2}}$ are tangent to $\tilde{M}$ in general only at the points of $\{r=0\}$. Of particular interest here will be the complex vector fields $Z_{+}=-\left(Z_{u_{2}}+i Z_{u_{1}}\right), Z_{-}=$ $-\left(Z_{u_{2}}-i Z_{u_{1}}\right)$. Since $H_{00}$, is tangent to $\tilde{M}$, complex vector fields $X_{+}, X_{-}$can be defined on $\tilde{M}$ by the condition

$$
X_{+}=Z_{+}, X_{-}=Z_{-} \text {at }\{r=0\}, \quad\left[H_{00^{\prime}}, X_{+}\right]=0, \quad\left[H_{00^{\prime}}, X_{-}\right]=0 \text { on } \tilde{M},
$$

where the commutators of complex vector fields and later the evaluation of forms on complex vector fields is defined by requiring complex linearity. From (4.1), (4.3)-(4.6) one finds

$$
\begin{aligned}
{\left[S, X_{+}\right] } & =2 X_{+}, \quad\left[S, X_{-}\right]=-2 X_{-}, \quad\left[X_{+}, X_{-}\right]=-S, \\
{\left[\partial_{u}, X_{+}\right] } & =0, \quad\left[\partial_{u}, X_{-}\right]=0 \text { on } \tilde{M} .
\end{aligned}
$$


There are different useful ways to look at the vector fields $X_{+}, X_{-}$. If considered as fields on $\tilde{M} \subset S(M)$ they are fields which are vertical at $\{r=0\}$, pick up a non-vanishing horizontal component for $r>0, r$ not too large (see (4.10)) and possibly become vertical again for sufficiently large $r$. In fact, in the case of example (1.1), (1.2) the fields $X_{+}, X_{-}$approach the fields $-Z_{+},-Z_{-}$near the point $i^{0}$. In the parametrization of $\tilde{M}$ by $u, r$ and $t$ the fields $S, X_{+}, X_{-}$may be regarded as complex linear combinations of the left invariant vector fields on $S U(2)$ determined by the generators (4.3). By the Hopf map $\pi^{\prime}$ of SU(2) onto $S^{2}$ they are related to the "edth-operator" and its complex conjugate of Newman and Penrose [13].

Any vector field on $\tilde{M}$ can now be represented as a linear combination of the fields $\partial_{u}, \partial_{r}, X_{+}, X_{-}, S$. On $\tilde{M}$ one has

$$
\left\langle\bar{\omega}_{b^{\prime}}^{a^{\prime}}, X_{+}\right\rangle=\left\langle\overline{\omega_{b}^{a}, X_{-}}\right\rangle,\left\langle\bar{\omega}_{b^{\prime}}^{a^{\prime}}, X_{-}\right\rangle=\left\langle\overline{\omega_{b}^{a}, X_{+}}\right\rangle,
$$

and in particular at $\{r=0\}$

$$
\begin{aligned}
& \left\langle\sigma^{a a^{\prime}}, X_{+}\right\rangle=0,\left\langle\omega^{a}{ }_{b}, X_{+}\right\rangle=\varepsilon_{0}{ }^{a} \varepsilon_{b}{ }^{1}, \\
& \left\langle\sigma^{a a^{\prime}}, X_{-}\right\rangle=0,\left\langle\omega^{a}{ }_{b}, X_{-}\right\rangle=-\varepsilon_{1}{ }^{a} \varepsilon_{b}{ }^{0} .
\end{aligned}
$$

From (4.2), (4.6), (4.8), (3.4) and the fact that the curvature form is horizontal ensues

$$
\begin{aligned}
& \left\langle\sigma^{a a^{\prime}}, X_{+}\right\rangle=-r \varepsilon_{0}{ }^{a} \varepsilon_{1}{ }^{a^{\prime}}+O\left(r^{2}\right), \\
& \left\langle\sigma^{a a^{\prime}}, X_{-}\right\rangle=-r \varepsilon_{1}{ }^{a} \varepsilon_{0^{\prime}}{ }^{{ }^{\prime}}+O\left(r^{2}\right), \\
& \left\langle\omega^{a}{ }_{b}, X_{+}\right\rangle=\varepsilon_{0}{ }^{a} \varepsilon_{b}{ }^{1}+O\left(r^{2}\right), \\
& \left\langle\omega^{a}{ }_{b}, X_{-}\right\rangle=-\varepsilon_{1}{ }^{a} \varepsilon_{b}{ }^{0}+O\left(r^{2}\right) .
\end{aligned}
$$

By construction the subspaces of the tangent spaces of $\tilde{M}$ which are spanned by the fields $\partial_{u}, \partial_{r}, X_{+}, X_{-}$are Lie propagated in the direction of $\partial_{r}$. From the definition of $u, r$ and from (4.10) follows that at a point $p \in \tilde{M}$ where $r>0$ but $r$ is not too large, the tangent space $T_{\tilde{\pi}(p)} M$ of $M$ is the image under $T(\tilde{\pi})$ of the 4dimensional subspace of $T_{p} \tilde{M}$ spanned by $\partial_{u}, \partial_{r}, X_{+}, X_{-}$. This implies that this subspace contains four uniquely determined vectors $e_{a a^{\prime}}, a, a^{\prime}=0,1$, satisfying $e_{a a^{\prime}}$ $=\bar{e}_{a a^{\prime}}$ and

$$
\left\langle\sigma^{b b^{\prime}}, e_{a a^{\prime}}\right\rangle=\varepsilon_{a}{ }^{b} \varepsilon_{a^{\prime}}^{b^{\prime}} .
$$

Formally the fields $e_{a a^{\prime}}$ are fixed uniquely by (4.11) and the requirements

$$
\begin{aligned}
& e_{00^{\prime}}=H_{00^{\prime}}=\partial_{r} \text { on } \tilde{M}, \\
& e_{11^{\prime}}=H_{11^{\prime}}=\partial_{u}-\partial_{r} \text { at }\{r=0\}, e_{11^{\prime}} \text { smooth on } \tilde{M}, \\
& e_{01^{\prime}}+\frac{1}{r} X_{+}, \quad e_{10^{\prime}}+\frac{1}{r} X_{-} \text {are smooth on } \tilde{M} \text { and vanish at }\{r=0\}, \\
& \quad\left[e_{00^{\prime}}, e_{a a^{\prime}}\right]=c_{00^{\prime}}{ }^{b b^{\prime}}{ }_{a a^{\prime}} e_{b b^{\prime}} \text { for } r>0, \text { not too large. }
\end{aligned}
$$

The conditions (4.12), motivated by the construction of $\tilde{M}$ and by (4.10), imply the correct limiting behaviour of the fields when approaching $\{r=0\}$, while (4.13), which requires that the commutators involve the fields $e_{a a^{\prime}}$ but not $S$, ensures that the fields 
$e_{a a^{\prime}}$ are in fact in the subspace spanned by $\partial_{u}, \partial_{r}, X_{+}, X_{-}$. Thus one has an expansion of the form

$$
\begin{aligned}
e_{a a^{\prime}}= & -\frac{1}{r}\left(\varepsilon_{a}{ }^{0} \varepsilon_{a^{\prime}}{ }^{1^{\prime}} X_{+}+\varepsilon_{a}{ }^{1} \varepsilon_{a^{\prime}}{ }^{{ }^{\prime}} X_{-}\right)+b_{a a^{\prime}} X_{+}+\bar{b}_{a a^{\prime}} X_{-} \\
& +\varepsilon_{a}{ }^{1} \varepsilon_{a^{\prime}}{ }^{\prime} \partial_{u}+\left(\varepsilon_{a}{ }^{0} \varepsilon_{a^{\prime}}{ }^{{ }^{\prime}}-\varepsilon_{a}{ }^{1} \varepsilon_{a^{\prime}}{ }^{{ }^{\prime}}\right) \partial_{r}+r_{a a^{\prime}} \partial_{r}
\end{aligned}
$$

with smooth functions $b_{a a^{\prime}}$ (not necessarily satisfying the reality condition) and $r_{a a^{\prime}}=\bar{r}_{a a^{\prime}}$ such that

$$
b_{00^{\prime}} \equiv 0, \quad r_{00^{\prime}} \equiv 0, \quad b_{a a^{\prime}}=O(r), \quad r_{a a^{\prime}}=O(r) .
$$

Equation (4.11) implies that for $u, r, t$ with $r>0, r$ not too large, one has

$$
T(\tilde{\pi}) e_{b b^{\prime}}(u, r, t)=c_{b b^{\prime}}(u, r, t) .
$$

The fields $e_{a a^{\prime}}, S$ constitute a frame near the set $\{r=0\} \cup\{u=0\}$, the points of $\{r=0\}$ excluded, with respect to which the field equations will be expressed.

The "connection coefficients" are obtained by applying the connection form to the fields $e_{a a^{\prime}}$. Because of (4.10), (4.12), (3.1) they are of the form

$$
\left\langle\omega_{b}^{a}, e_{c c^{\prime}}\right\rangle=-\frac{1}{r} \Gamma_{c c^{\prime} b}^{* a}+\Gamma_{c c^{\prime} b}^{a}
$$

with smooth functions $\Gamma_{c c^{\prime}{ }_{b}}{ }^{a}$ and

$$
\begin{aligned}
\Gamma_{c c^{\prime} b}^{* a} & =\varepsilon_{c}{ }^{0} \varepsilon_{c^{\prime}}{ }^{\prime} \varepsilon_{0}{ }^{a} \varepsilon_{b}{ }^{1}-\varepsilon_{c}{ }^{1}{\varepsilon_{c^{\prime}}}{ }^{\prime} \varepsilon_{1}{ }^{a} \varepsilon_{b}{ }^{0}, \\
\Gamma_{00^{\prime}{ }_{b}} & \equiv 0, \Gamma_{c c^{\prime} b}{ }^{a}=O(r) .
\end{aligned}
$$

It may be pointed out here that the meaning of the quantities $\Gamma_{a a^{\prime}{ }_{c}}$ used here differs from that of the quantities denoted in $[5,7]$ by the same symbols.

\section{The Dependence of the Fields on the Angular Coordinates}

To describe the regularity conditions on the fields near $\{r=0\}$, i.e. of their behaviour near the vertices of the cones $N_{\gamma(u)}$, a thorough analysis of their dependence on the variable $t \in \mathrm{SU}(2)$ is required. By choosing a local trivialization of the Hopf fibration $\pi^{\prime}: \operatorname{SU}(2) \rightarrow S^{2}$, as realized by $\delta(u, 0, t) \rightarrow c_{00^{\prime}}(u, 0, t)$ and by introducing coordinates on the base space $S^{2}$ and a parametrization of $U(1)$ of the form $\mathbb{R} \ni \phi \rightarrow \exp (\phi \cdot h), h$ as in (4.3), a local coordinate system is obtained on $\mathrm{SU}(2)$.

The dependence of the fields on $M$ on the fibre coordinate $\phi$ reflects their transformation properties on the fibres of $S(M)$. It is readily described by their behaviour under the action of the vector field $S$. A complex-valued function $f=f(u, r, t)$ on $M$ (respectively $f=f(t)$ on $\mathrm{SU}(2))$ will be called to be of spin weight $s$, if

$$
S f=2 s f \quad \forall t \in \mathrm{SU}(2) \text {. }
$$

As will be seen later, all unknown which appear in the fields equations if they are expressed with respect to the frame $e_{a a^{\prime}}, S$ possesses a well defined integer spinweight. 
The dependence of a spinor field on the variable $r$ and the coordinates on $S^{2}$, i.e. on the angular coordinates on $N_{\gamma(u)}$, may be studied by introducing normal coordinates based on the point $\gamma(u)$ and an orthonormal frame at $\gamma(u)$ with a timelike vector proportional to $\delta^{a a^{\prime}} c_{a a^{\prime}}(u, 0, t)$. The coefficients of the Taylor expansion of a field in terms of the normal coordinates are given by the covariant derivatives of the field at $\gamma(u)$. Restriction of this expansion to $N_{\gamma(u)}$ yields an expansion in terms of $r$ with expansion coefficients, which reflect the dependence on the angular variables [14]. By suitable contractions with $\delta_{a}{ }^{a^{\prime}}$ and decomposition into irreducible parts, the expansion coefficients on $N_{\gamma(u)}$ are finally obtained as complicated sums of spinors of the type

$$
\phi_{a_{1} \ldots a_{n}}=\phi_{\left(a_{1} \ldots a_{n}\right)},
$$

which are contracted with $t_{0}^{a_{1}} \cdots t_{0}^{a_{j}} t_{1}^{a_{j}+1} \cdots t_{1}^{a_{2 j}}$ for some appropriate $j \leqq n$. In the following a somewhat similar expansion will be studied on $\tilde{M}$ and it will be seen that it amounts to expanding functions on $\tilde{M}$ in terms of a complete function system on $\mathrm{SU}(2)$ which arises naturally in the present setting.

Let $t \rightarrow t^{a}{ }_{b}$ denote as before the 2-dimensional standard representation of the group SL $(2, \mathbb{C})$ and set $t_{\alpha}^{(\beta)}=t_{a_{1}}^{\left(b_{1}\right.} \cdots t_{a_{m}}^{\left.b_{m}\right)}$ such that $\alpha, \beta$ denote multi-indices of length $m$ and $(\beta)$ symmetrization of the indices indicated by $\beta$. Furthermore let $\alpha_{k}$ for some $k$, $0 \leqq k \leqq m$, denote the evaluation of the multi-index $\alpha$ by setting the first $k$ indices equal to 1 and the last $m-k$ indices equal to 0 . In the following spinor valued functions $\phi^{\alpha}=\phi^{a_{1} \ldots a_{m}}=\phi^{\left(a_{1} \ldots a_{m}\right)}$ with "essential components" $\phi^{k}=\phi^{\alpha_{k}}$ will be considered. Identifying a certain point $p \in S(M)$ (this will later be the point $\delta(u, 0,1), 1$ the unit element in $\mathrm{SU}(2))$ with the unit element in $\operatorname{SL}(2, \mathbb{C})$, the function implied by $\phi^{\alpha}$ on the fibre $\bar{\pi}^{1}(\pi(p))$ can be written in a unique way as a linear combination with constant coefficients of the functions

$$
t \rightarrow T_{m}{ }_{k}{ }_{k}(t)=\left(\begin{array}{c}
m \\
j
\end{array}\right)^{1 / 2}\left(\begin{array}{c}
m \\
k
\end{array}\right)^{1 / 2} t^{(\beta) j}{ }_{\alpha_{k}}, \quad j, k=0, \ldots, m
$$

on $\operatorname{SL}(2, \mathbb{C})$. The factors of $t^{(\beta)}{ }_{\alpha}$ are suggested by the following consideration. To the transformation (2.1) of a spinor-valued function under the action of $\operatorname{SL}(2, \mathbb{C})$ on the fibres of $S(M)$ corresponds a representation $\operatorname{SL}(2, \mathbb{C}) \ni t \rightarrow T(t) \in \operatorname{End}\left(S^{m}\right)$ with

$$
T(t): \phi^{\alpha} \rightarrow t_{\beta}^{(\alpha)} \phi^{\beta}
$$

of the group $\operatorname{SL}(2, \mathbb{C})$ on the linear space $S^{m}$ of spinors of valence $m$. On this space a hermitian scalar product is defined by

$$
(\phi, \psi)=\phi^{a_{1} \ldots a_{m}} \Psi^{a_{1}^{\prime} \cdot a_{m}^{\prime}} \delta_{a_{1} a_{1}^{\prime}} \cdots \delta_{a_{m} a_{m}^{\prime}} .
$$

An orthonormal basis for this scalar product is given by the spinors

$$
\phi_{j}^{\alpha}=\left(\begin{array}{c}
m \\
j
\end{array}\right)^{1 / 2} \varepsilon_{\left(b_{1}\right.}{ }^{a_{1}} \cdots \varepsilon_{\left.b_{m}\right) j} a_{m}, \quad j=0, \ldots, m .
$$

The matrix elements of the transformation $T(t)$ with respect to this basis are given by the functions $T_{m}{ }_{k}(t)$ of (5.2). Because of (3.3) the restriction of the representation $T$ of $\mathrm{SL}(2, \mathbb{C})$ to $\mathrm{SU}(2)$ leaves invariant the scalar product $(5.4)$. Thus $(5.2)$ provides the 
matrix elements with respect to an orthonormal basis of a $(m+1)$-dimensional continuous unitary representation

$$
\mathrm{SU}(2) \ni t \rightarrow T_{m}(t)=\left(T_{m k}^{i}(t)\right) \in \mathrm{SU}(m+1),
$$

where $T_{0}$ denotes the trivial representation. It is well known that the set of representations $T_{m}, m \in \mathbb{N}$ constitutes a complete set of irreducible unitary representations of the group SU(2). By the Peter-Weyl theorem the functions $\sqrt{m+1} T_{m}{ }_{k}(t)$ with $m \in \mathbb{N}, i, k=0, \ldots, m$, form a complete orthonormal set in the space $L_{2}(\mu$, $\mathrm{SU}(2)$ ), where $\mu$ is the normalized Haar measure on SU(2). Thus an expansion in terms of these functions will be quite general. All the other properties of the functions $T_{m k}{ }^{i}$ used in this paper and in the investigation of the analytic case can be deduced from the explicit expression (5.2) and from the fact that (5.6) constitutes an analytic representation. As an example one gets the relations

$$
\overline{T_{m}{ }^{i}(t)}=(-1)^{i+k} T_{m}{ }^{m-i}{ }_{m-k}(t) \quad \forall t \in \mathrm{SU}(2)
$$

from (3.3), and the relation $\varepsilon_{a b} t^{a} t^{b}{ }_{d}=\varepsilon_{c d}$ which is satisfied by all $t \in \mathrm{SU}(2)$. Setting $p=\delta(u, 0,1)$ and identifying the fields $X_{+}, X_{-}, S$ on $\tilde{\pi}^{-1}(\gamma(u))$ with left invariant vector vector fields on $\mathrm{SU}(2)$, one finds on that set

$$
\begin{aligned}
S T_{m j}{ }^{k} & =(m-2 j) T_{m j}{ }^{k}, \\
X_{+} T_{m j}{ }^{k}{ }_{j} & =\beta_{m, j} T_{m j-1}{ }^{k}, \quad \forall m \in \mathbb{N}, \quad k, j=0, \ldots, \\
X_{-} T_{m j}{ }^{k} & =-\beta_{m, j+1} T_{m j+1}{ }^{k}, \quad \text { with } \quad \beta_{m, j}=\{j(m-j+1)\}^{1 / 2} .
\end{aligned}
$$

In the parametrization of $\tilde{M}$ by $u, r, t$ these relations hold everywhere on $\tilde{M}$, if the functions $T_{m}{ }^{k}$ are defined for $r>0$ by dragging them along with $\partial_{r}$.

Let $f$ be a smooth function on $\tilde{\pi}^{-1}(\gamma(u))$ such that

$$
S f=2 s f, \quad X_{+}^{n} f=0 \quad \forall n \geqq n_{0}+1,
$$

and assume that $s \in \mathbb{Z}$, since only this case will be considered later. From (5.8) follows that the expansion of $f$ in terms of $T_{m}{ }_{l}{ }_{l}$ can only contain those functions for which $m-2 l=2 s$ and $l \leqq n_{0}$. Unless $f$ vanishes identically, one must thus have $n_{0} \geqq|s|-s$ and the expansion of $f$ must be of the form

$$
f=\sum_{n=0}^{n_{0}+s-|s|} \sum_{k=0}^{2(n+|s|)} f_{n, k} T_{2(u+|s|)_{n+|s|-s}}^{k}
$$

with $f_{n, k} \in \mathbb{C}$.

Suppose $\phi_{\alpha \beta^{\prime}}=\phi_{(\alpha)\left(\beta^{\prime}\right)}=\phi_{a_{1} \ldots a_{p} b_{1}^{\prime} \ldots b_{q}^{\prime}}$ is a spinor valued function on $S(M)$ and denote by $\phi_{j k}=\phi_{\alpha_{j} \beta_{k}}, 0 \leqq j \leqq p, 0 \leqq k \leqq q$, the essential components. The purpose of the following discussion is to exhibit the analogue of the expansion (5.10) for the functions obtained on $\tilde{\pi}^{-1}(\gamma(u))$ by restricting $H_{00}{ }^{l} \phi_{j k}, l \in \mathbb{N}$, to this set.

Defining $\phi_{j k}$ to be equal to zero if $j, k<0$ or $j>p$ or $k>q$, the relations

$$
\begin{aligned}
S \phi_{j k} & =(p-q+2(j-k)) \phi_{j k}, \\
Z_{+} \phi_{j k} & =j \phi_{j-1, k}-(q-k) \phi_{j, k+1}, \\
Z_{-} \phi_{j k} & =-(p-j) \phi_{j+1, k}+k \phi_{j, k-1}
\end{aligned}
$$


hold everywhere on $S(M)$. They may be derived in a similar way as (5.8) by using an expansion in terms of the functions $T_{m}{ }_{i}{ }_{i}$ and using (5.7). Specializing to the set $\tilde{\pi}^{-1}(\gamma(u))$ one finds that there the relations (5.11)-(5.13) hold with $Z_{+}, Z_{-}$being replaced by $X_{+}, X_{-}$. In particular it follows from these relations that the functions $\phi_{j k}$ can be calculated on $\tilde{\pi}^{-1}(\gamma(u))$ by $X_{+}, X_{-}$-operations if $\phi_{00}$ is given there.

By iterated application of $Z_{+}$to the first of Eqs. (5.12) one finds

$$
Z_{+}^{n} \phi_{j k}=0 \quad \forall n \geqq n_{0}=j-q-k+1
$$

and in general $n_{0}$ is the smallest such integer. Again $Z_{+}$may be replaced by $X_{+}$on $\tilde{\pi}^{-1}(\gamma(u))$. From (4.1), (4.3) ensues

$$
\begin{aligned}
& {\left[Z_{+}, H_{00^{\prime}}\right]=-H_{01^{\prime}}, \quad\left[Z_{+}, H_{01^{\prime}}\right]=0,} \\
& {\left[Z_{+}, H_{00^{\prime}}+H_{11^{\prime}}\right]=0 \quad \text { on } S(M),}
\end{aligned}
$$

whence by induction

$$
Z_{+}^{l+n} H_{00^{\prime}}{ }^{l}=\left(H_{00^{\prime}}{ }^{l} Z_{+}{ }^{l}+\sum_{i=1}^{l-1} \hat{H}_{i} Z_{+}^{l-i}+c H_{01^{\prime}}{ }^{l}\right) Z_{+}{ }^{n}
$$

for $l, n \in \mathbb{N}$ with $l \geqq 1, n \geqq 0$. Here $\hat{H}_{i}$ denotes some operator on $S(M), c$ a nonvanishing real number, both depending on $l, n$. Using (5.14), (5.16) one concludes that on $\tilde{\pi}^{-1}(\gamma(u))$,

$$
X_{+}^{l+n}\left(\partial_{r}^{l} \phi_{j k}\right)=Z_{+}^{l+n} H_{00^{\prime}}{ }^{l} \phi_{j k}=0, \quad \forall l \geqq 0 \quad \forall n \geqq n_{0}=j+q-k+1 .
$$

Furthermore (4.4) gives

$$
S\left(\partial_{r}^{l} \phi_{j k}\right)=H_{00^{\prime}}^{l} S \phi_{j k}=(p-q-2(j-k)) \phi_{j k} \quad \forall l \geqq 0 .
$$

Comparison with (5.9), (5.10) yields the expansion for $\partial_{r}^{l} \phi_{j k}$ on $\tilde{\pi}^{-1}(\gamma(u))$. It follows that the restriction of $\phi_{j k}$ to $\tilde{M}$ must have a Taylor expansion at $\{r=0\}$ of the form

$$
\left.\begin{array}{rl}
\phi_{j k} & =\sum_{l=0}^{N} \phi_{j k, l} r^{l}+O\left(r^{N+1}\right) \quad \text { for } N \in \mathbb{N}, \text { with } \\
\phi_{j k, l} & =\sum_{n=0}^{l+d} \sum_{i=0}^{2(n+|s|)} \phi_{j k, l, n, i} T_{2(n+|s|)_{n+|s|-s}^{i}}^{i}
\end{array}\right\}
$$

with $s=\frac{1}{2}(p-q)-(j-k), d=j+q-k+s-|s|$, and coefficients $\phi_{j k, l, n, i}$, which are smooth functions of $u$. The last of Eqs. (5.15) implies as to be expected, that there is no particular relation between the dependence on $u$ and on $t$. The remark following (5.12) shows that the quantities $\phi_{j k, 0}$ can be calculated by $X_{+}, X_{-}$-operations from $\phi_{00,0}$.

The expansion (5.19) is characterized by two numbers: the spin weight $s$ and the integer $d$. For the frame coefficients $r_{a a^{\prime}}, b_{a a^{\prime}}$ and the connection coefficients $\Gamma_{a a^{\prime} c}{ }_{c}$ on $\tilde{M}$ near $\{r=0\}$ expansions in terms of $r$ and the $T_{m}{ }^{i} k$ cannot be discussed in this way. However, it can be shown that the field Eqs. (6.4)-(6.7) together with the conditions on the data imply expansions for these coefficients which are of the same form as (5.19) and again characterized by $s$ and $d$. For the unknowns in the field Eqs. (6.4)-(6.7) the numbers $s$ and $d$ can be read off from the following table: 
Table 1. It is here understood that in (5.19) coefficients $\phi_{j k, l, n, i}$ are defined to be zero if $l$ or $n$ are negative. For the definition of $c_{a a^{\prime}}, \gamma_{a a^{\prime}}$ and the discussion of the spin weight see the next chapter

\begin{tabular}{lllll}
\hline & $d=-1$ & $d=0$ & $d=1$ & $d=2$ \\
\hline$s=-2$ & $c_{10^{\prime}}, \gamma_{10^{\prime}}$ & $\varphi_{4}, \phi_{20}$ & & \\
\hline$s=-1$ & $r_{10^{\prime}}$ & $\Gamma_{10^{\prime} 01}, c_{11^{\prime}}, \gamma_{11^{\prime}}$ & $\varphi_{3}, \phi_{10}, \phi_{21}$ & \\
\hline$s=0$ & & $r_{11^{\prime}}, \Omega, s, \Lambda$ & $\Gamma_{10^{\prime} 00}, c_{01^{\prime}}, \gamma_{01^{\prime}}$ & $\Gamma_{11^{\prime} 01}, \Sigma_{00^{\prime}}, \Sigma_{11^{\prime}}$ \\
& & & $\varphi_{1}, \phi_{01}, \phi_{12}$ & \\
\hline$s=1$ & $r_{01^{\prime}}$ & $\Gamma_{01^{\prime} 01}, \Gamma_{11^{\prime} 00}, \Sigma_{01^{\prime}}$ & \\
\hline$s=2$ & $\Gamma_{01^{\prime} 00}$ & $\varphi_{0}, \phi_{02}$ & & \\
\hline
\end{tabular}

\section{The Field Equations on $\tilde{M}$}

The equations of forms implied by (2.3)-(2.11) on $\tilde{M}$ have to be expressed in terms of a suitably chosen frame to obtain a representation of the equation as a system of partial differential equations. Since the frame $\partial_{u}, \partial_{r}, X_{+}, X_{-}, S$ is globally defined and smooth on $\tilde{M}$, one may wonder whether it is necessary at all to introduce the fields $e_{a a^{\prime}}$, which become singular on the caustic set. The reason is that the symmetry requirements $\varphi_{a b c d}=\varphi_{(a b c d)}, \phi_{a b a^{\prime} b^{\prime}}=\phi_{(a b)\left(a^{\prime} b^{\prime}\right)}$ form an essential part of the content of the field equations. If these symmetries are not made explicit in some formalism, Eq. (2.5) does not contain more information than the Bianchi identity $D \Omega^{a}{ }_{b}=0$ for the curvature form.

Expressing Eqs. (2.3)-(2.11) in terms of the frame $e_{a a^{\prime}}, S$ one obtains two sets of equations. Evaluations involving the vector field $S$ simply give relations which determine the spin weights of the unknowns, while evaluations involving only the fields $e_{a a^{\prime}}$ give the propagation equations.

Equation (2.3) evaluated on $S \wedge e_{b b^{\prime}}$ gives

$$
\left\langle\sigma^{a a^{\prime}},\left[S, e_{b b^{\prime}}\right]\right\rangle=\left\langle\omega_{b}^{a}, S\right\rangle \varepsilon_{b^{\prime}} a^{\prime}+\left\langle\bar{\omega}^{a^{\prime}}{ }_{b^{\prime}}, S\right\rangle \varepsilon_{b}{ }^{a}
$$

By (4.4), (4.7), (4.13) the commutator $\left[S, e_{b b^{\prime}}\right]$ can be expressed in terms of the fields $e_{a a^{\prime}}$. Therefore (4.4), (6.1) imply

$$
\left[S, e_{b b^{\prime}}\right]=2\left(\varepsilon_{b}{ }^{0} \varepsilon_{b^{\prime}}{ }^{\prime} e_{01^{\prime}}-\varepsilon_{b}{ }^{1} \varepsilon_{b^{\prime}}{ }^{0^{\prime}} e_{10^{\prime}}\right) \text {. }
$$

For the coefficients of $e_{a a^{\prime}}$ in the expression (4.14) this entails spin weights as given in Table 1. Evaluation of (2.4) on $S \wedge e_{c c^{\prime}}$ gives

$$
S\left\langle\omega_{b}^{a}, e_{c c^{\prime}}\right\rangle-\left\langle\omega_{b}^{a},\left[S, e_{c c^{\prime}}\right]\right\rangle=\left\langle\omega_{d}^{a}, S\right\rangle\left\langle\omega_{b}^{d}, e_{c c^{\prime}}\right\rangle+\left\langle\omega_{d}^{a}, e_{c c^{\prime}}\right\rangle\left\langle\omega_{b}^{d}, S\right\rangle .
$$

Observing (4.17), (4.18) one finds that all the terms $\Gamma_{a a^{\prime} c}^{* b}$ drop out of this equation, which reduces to a relation giving the spin weights of the functions $\Gamma_{a a^{\prime}{ }^{b} c}$ as listed in Table 1. In a similar way one obtains the spin weights for the other fields from the remaining equations in accordance with formula (5.11).

Only that part of the propagation equations will be derived here by evaluation 
of (2.3)-(2.11) on the fields $e_{a a^{\prime}}$, which is essential for the following argument. It turns out that the structure of the singular part of the equations becomes particularly simple if the following new unknowns are introduced:

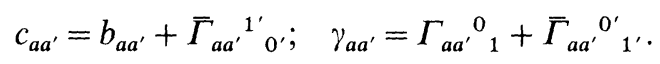

Furthermore it is convenient to use the notation

$$
e_{a a^{\prime}}=-\frac{1}{r}\left(\varepsilon_{a}{ }^{0} \varepsilon_{a^{\prime}}{ }^{\prime} X_{+}+\varepsilon_{a}{ }^{1} \varepsilon_{a^{\prime}}{ }^{0^{\prime}} X_{-}\right)+e_{a a^{\prime}}^{*}
$$

to display clearly the singular part of the equations.

The following interior equations are implied by (2.3)-(2.11) on the hypersurfaces $\tilde{N}_{u}$ of $\tilde{M}$ :

$$
\begin{aligned}
& \partial_{r} r_{a a^{\prime}}+\frac{1}{r}\left(\varepsilon_{a}{ }^{0} \varepsilon_{a^{\prime}}{ }^{\prime} r_{01^{\prime}}+\varepsilon_{a}{ }^{1} \varepsilon_{a^{\prime}}{ }^{0^{\prime}} r_{10^{\prime}}\right) \\
& =\bar{\Gamma}_{a a^{\prime} 0^{\prime} 0^{\prime}} r_{01^{\prime}}+\Gamma_{a a^{\prime} 00^{\prime}} r_{10^{\prime}}-\Gamma_{a a^{\prime} 10}-\bar{\Gamma}_{a a^{\prime} 1^{\prime} 0^{\prime}}, \\
& \partial_{r} c_{a a^{\prime}}+\frac{1}{r}\left(\varepsilon_{a}{ }^{0} \varepsilon_{a^{\prime}}{ }^{\prime} c_{01^{\prime}}+\varepsilon_{a}{ }^{1} \varepsilon_{a^{\prime}}{ }^{0^{\prime}} c_{10^{\prime}}\right) \\
& =\bar{\Gamma}_{a a^{\prime} 0^{\prime} 0^{\prime}} c_{01^{\prime}}+\Gamma_{a a^{\prime} 00^{\prime}} c_{10^{\prime}}+\bar{\Omega} \bar{\varphi}_{a^{\prime} 0^{\prime} 0^{\prime} 0^{\prime}} \varepsilon_{a 0}+\phi_{a 00^{\prime} 0^{\prime}} \varepsilon_{a^{\prime} 0^{\prime}}, \\
& \partial_{r} \Gamma_{c c^{\prime} 00}+\frac{1}{r}\left\{\varepsilon_{c}{ }^{0} \varepsilon_{c^{\prime}}{ }^{1^{\prime}} \Gamma_{01^{\prime} 00}+\varepsilon_{c}{ }^{1} \varepsilon_{c^{\prime}}{ }^{0^{\prime}} \Gamma_{10^{\prime} 00}+\Gamma_{c c^{\prime} 00}\right\} \\
& =\bar{\Gamma}_{c c^{\prime} 0^{\prime} 0^{\prime}} \Gamma_{01^{\prime} 00}+\Gamma_{c c^{\prime} 00} \Gamma_{10^{\prime} 00}-\Omega \varphi_{c 000^{\prime}} \varepsilon_{c^{\prime} 0^{\prime}}-\phi_{00 c^{\prime} 0^{\prime}} \varepsilon_{c 0}, \\
& \partial_{r} \Gamma_{c c^{\prime} 01}+\frac{1}{r}\left\{\varepsilon_{c}^{0} \varepsilon_{c^{\prime}}{ }^{\prime} \Gamma_{01^{\prime} 01}+\varepsilon_{c}{ }^{1} \varepsilon_{c^{\prime}}{ }^{0^{\prime}} \Gamma_{10^{\prime} 01}\right\} \\
& =\bar{\Gamma}_{c c^{\prime} 0^{\prime} 0^{\prime}} \Gamma_{01^{\prime} 01}+\Gamma_{c c^{\prime} 00} \Gamma_{10^{\prime} 01}-\Omega \varphi_{c 001} \varepsilon_{c^{\prime} 0^{\prime}}-\phi_{01 c^{\prime} 0^{\prime}} \varepsilon_{c 0}-\Lambda \varepsilon_{c^{\prime} 0^{\prime}} \varepsilon_{0 c} \\
& \partial_{r} \gamma_{a a^{\prime}}+\frac{1}{r}\left\{\varepsilon_{a}{ }^{0} \varepsilon_{a^{\prime}}{ }^{\prime} \gamma_{01^{\prime}}+\varepsilon_{a}{ }^{1} \varepsilon_{a^{\prime}}{ }^{0^{\prime}} \gamma_{10^{\prime}}\right\} \\
& =\bar{\Gamma}_{a a^{\prime} 0^{\prime} 0^{\prime}} \gamma_{01^{\prime}}+\Gamma_{a a^{\prime} 00} \gamma_{10^{\prime}}-\Omega \varphi_{a 011} \varepsilon_{a^{\prime} 0^{\prime}}+\bar{\Omega} \bar{\varphi}_{a^{\prime} 0^{\prime} 0^{\prime} 0^{\prime}} \varepsilon_{a 0} \\
& -\phi_{11 a^{\prime} 0^{\prime}} \varepsilon_{a 0}+\phi_{a 00^{\prime} 0^{\prime}} \varepsilon_{a^{\prime} 0^{\prime}}+2 \Lambda \varepsilon_{a 1} \varepsilon_{a^{\prime} 0^{\prime}} \\
& \partial_{r} \varphi_{a b c 1}+\frac{1}{r}\left\{X_{-}\left(\varphi_{a b c 0}\right)+3 \varepsilon_{(a}{ }^{0} \varphi_{b c) 01}+\varphi_{a b c 1}\right\}-e_{10^{\prime}}^{*}\left(\varphi_{a b c 0}\right) \\
& =-3 \Gamma_{10^{\prime}{ }_{(a}^{e} \varphi_{b c) e 0}}-\Gamma_{10^{\prime}{ }_{0}}^{e} \varphi_{a b c e}, \\
& \partial_{r} \phi_{b c d^{\prime} 1^{\prime}}+\frac{1}{r}\left\{X_{+}\left(\phi_{b c d^{\prime} 0^{\prime}}\right)+\varepsilon_{d^{\prime}}{ }^{0^{\prime}} \phi_{b c 1^{\prime} 0^{\prime}}+\phi_{b c d^{\prime} 1^{\prime}}-2 \varepsilon_{(b}{ }^{1} \phi_{c) 0 d^{\prime} 0^{\prime}}\right\}-e_{01^{\prime}}^{*}\left(\phi_{b c d^{\prime} 0^{\prime}}\right) \\
& =-2 \Gamma_{01}{ }^{e}{ }_{(b} \phi_{c) e d^{\prime} 0^{\prime}}-\bar{\Gamma}_{01^{\prime}}{ }^{e^{\prime}{ }_{d^{\prime}}} \phi_{b c e^{\prime} 0^{\prime}}
\end{aligned}
$$

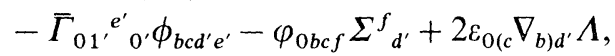

$$
\begin{aligned}
& \partial_{r} \Omega=\Sigma_{00^{\prime}} \\
& \partial_{r} \Sigma_{b b^{\prime}}=-\Omega \phi_{0 b 0^{\prime} b^{\prime}}+s \varepsilon_{0 b^{\prime}} \varepsilon_{0^{\prime} b^{\prime}}, \\
& \partial_{r} s=-\phi_{0 b 0^{\prime} b^{\prime}} \Sigma^{b b^{\prime}}-2 \Lambda \Sigma_{00^{\prime}}+\Omega \partial_{r} \Lambda,
\end{aligned}
$$




$$
2 \Omega_{S}-\Sigma_{a a^{\prime}} \Sigma^{a a^{\prime}}+2 \Omega^{2} \Lambda=0 .
$$

Of those equations which involve derivatives in the direction of $e_{11}$, transverse to the hypersurfaces $\tilde{N}_{u}$, only the following are needed.

$$
\begin{aligned}
& \nabla_{11^{\prime}}, \varphi_{0000}+\frac{1}{r}\left\{X_{+}\left(\varphi_{0001}\right)-\varphi_{0000}\right\}-e_{01}^{*}\left(\varphi_{0001}\right) \\
& =-3 \Gamma_{01}{ }^{e}{ }_{0} \varphi_{e 001}-\Gamma_{01}{ }^{e}{ }_{1} \varphi_{000 e} \\
& \nabla_{11} \phi_{000^{\prime} 0^{\prime}}+\frac{1}{r}\left\{X_{-}\left(\phi_{000^{\prime} 0^{\prime}}\right)+2 \phi_{010^{\prime} 1^{\prime}}-\phi_{000^{\prime} 0^{\prime}}\right\}-e_{10^{\prime}}^{*}\left(\phi_{000^{\prime} 1^{\prime}}\right)
\end{aligned}
$$

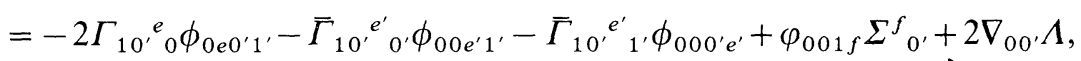

$$
\begin{aligned}
& \nabla_{11^{\prime}} \Omega=\Sigma_{11^{\prime}} \\
& \nabla_{11^{\prime}} \Sigma_{c c^{\prime}}=-\Omega \phi_{1 c 1^{\prime} c^{\prime}}+S \varepsilon_{1 c^{\prime}} \varepsilon_{1^{\prime} c^{\prime}} \\
& \nabla_{11^{\prime}} s=-\phi_{1 b 1^{\prime} b^{\prime}} \Sigma^{b b^{\prime}}-2 \Lambda \Sigma_{11^{\prime}}-\Omega \nabla_{11^{\prime}} \Lambda \text {. }
\end{aligned}
$$

\section{Discussion of the Remaining Gauge Freedom}

The construction of the manifold $\tilde{M}$ was based on two arbitrary choices. From a class of conformally related metric field on $M$ which are compatible with the conditions (1.4), (1.5), (1.6) has been picked one particular field $g$ and in the fibre over $i^{-}$of the bundle of normalized spin frames with respect to $g$ has been chosen arbitrarily the spin frame $\left(\delta_{a}\right)$. Therefore it is of interest to know how the structures introduced so far change under a rescaling of the metric and a rotation of the spin frame. Particularly important is to understand the transformation behaviour of the initial data for the initial value problem which will be discussed in the next chapter.

Assume the metric $g$ has been chosen and the spin frame $\delta_{a}$ is transformed according to

$$
\delta_{a} \rightarrow \delta_{a}^{\prime}=\delta_{b} l_{a}^{b}
$$

with some $l_{b}^{a} \in \operatorname{SL}(2, \mathbb{C})$. Denote by $\tilde{M}^{\prime}, u^{\prime}, \ldots$ etc. all manifolds, functions etc. constructed with respect to $\delta_{a}^{\prime}$ as described in Chap. 3. Both the hypersurface $\tilde{N}_{0}$ as well as the hypersurface $\tilde{N}_{0}^{\prime}$ are mapped by the projection of $S(M)$ onto the cone $I_{l^{-}}$. The point $\delta(0, r, t) \in N_{0}, r>0$, is mapped onto the point on the null geodesics through $i^{-}$with tangent vector $c_{00^{\prime}}(0, r, t)$ with affine parameter distance $r$ from $i^{-}$. A similar remark holds for the quantities marked with a stroke. In order that $\delta(0, r, t)$ and $\delta^{\prime}\left(0, r^{\prime}, t^{\prime}\right)$ are in the same fibre of $S(M)$ one must have

$$
l_{b}^{a} t^{\prime b}{ }_{c}=t^{a}{ }_{b} d^{b}
$$

with some $d^{a}{ }_{b} \in \operatorname{SL}(2, \mathbb{C})$. Since both points project onto the same null geodesic it holds

$$
l_{b}^{a} t^{\prime}{ }_{0}=\lambda t^{a}{ }_{0}
$$

with $\lambda \in \mathbb{C}^{*}$. This implies

$$
c_{0 c^{\prime}}^{\prime}\left(0, r^{\prime}, t^{\prime}\right)=|\lambda|^{2} c_{0 c^{\prime}}(0, r, t)
$$


Because of the fibre structure of $\tilde{M}$ and $\tilde{M}^{\prime}$ there is still the freedom of replacement $\lambda \rightarrow e^{i \varphi} \lambda$ with some $\varphi \in \mathbb{R}$. This can be removed by requiring that not only the image points of $\delta(0, r, t), \delta^{\prime}\left(0, r^{\prime}, t^{\prime}\right)$ under the projection $\pi$ are the same, but that also the null flags [18] determined by the spinors $\delta_{0}(0, r, t)$. $\delta_{0}^{\prime}\left(0, r^{\prime}, t^{\prime}\right)$ coincide and that the transformation $d^{a}{ }_{b}=d^{a}{ }_{b}(t, l)$ depends continuously on $t$ and $l$ and approaches the unit element of $\operatorname{SL}(2, \mathbb{C})$, if $l$ does. Then $d^{a}{ }_{b}$ must be of the form

$$
d^{a}{ }_{b}=\left(\begin{array}{cc}
\lambda & \lambda^{-1} z \\
0 & \lambda^{-1}
\end{array}\right), \quad \lambda \in \mathbb{R}^{+}, \quad z \in \mathbb{C} .
$$

From (7.3), (7.4) ensue the transformation laws $t^{\prime}=t^{\prime}(t, r), r^{\prime}=r(t, r)$, given by

$$
r^{\prime}=\lambda^{-2} r \quad t^{\prime a}{ }_{0}=\lambda l^{-1 a} t^{b}{ }_{0} \quad \text { with } \quad \lambda=\left\|l^{-1 a} t^{b}{ }_{0}\right\|^{-1} \text {. }
$$

Here the norm is that implied by the product (5.4) and it may be noted that the transformation $t_{b}^{a} \in \mathrm{SU}(2)$ is uniquely determined by $t^{a}{ }_{0}$, since $t^{a}{ }_{1}=-\delta^{a}{ }_{f^{\prime}} \bar{t}^{f^{\prime}}{ }_{0^{\prime}}$. The function $z$ is also fixed uniquely, but will not be needed in the following. The coordinate transformation (7.6) and the fibre transformation (7.5) allow one to write down the relation between the functions implied on $\tilde{N}_{0}$ and $\tilde{N}_{0}^{\prime}$ by the functions $\varphi_{a b c d}, \phi_{a b a^{\prime} b^{\prime}}$ on $S(M)$. The only components of those fields which have homogeneous transformation laws under (7.5) are $\varphi_{0000}$ and $\phi_{000^{\prime} 0^{\prime}}$, which provide the initial data for the pure radiation problem. The corresponding functions on $\tilde{N}_{0}$ and $\tilde{N}_{0}^{\prime}$ are related by

$$
\begin{aligned}
\varphi_{0000}^{\prime}\left(0, r^{\prime}, t^{\prime}\right) & =\lambda^{4}(t) \varphi_{0000}(0, r, t), \\
\phi_{000^{\prime} 0^{\prime}}^{\prime}\left(0, r^{\prime}, t^{\prime}\right) & =|\lambda(t)|^{4} \phi_{000^{\prime} 0^{\prime}}(0, r, t) .
\end{aligned}
$$

To end the discussion of the transformations implied by (7.1) it may be remarked that the fibre structure of the bundle $S(M)$ does not imply a globally defined bijection of $\tilde{M}$ onto $\tilde{M}^{\prime}$ which commutes with the action of the group $U(1)$ on these two manifolds. However, there exists a neighbourhood $W$ of $\tilde{N}_{0} \backslash\{r=0, u=0\}$ in $\tilde{M}$, having empty intersection with the caustic set of $\tilde{M}$, on which such a map $f$ onto a similar neighbourhood $W^{\prime}$ of $\tilde{N}_{0}^{\prime} \backslash\left\{u^{\prime}=0, r^{\prime}=0\right\}$ can be defined. The map $f$ is fixed uniquely by the requirement that it extends the map of $\tilde{N}_{0}$ onto $\tilde{N}_{0}^{\prime}$ constructed above and that $T(f) S=S^{\prime}, T(f) e_{a a^{\prime}}=e^{\prime}{ }_{b b} s^{b}{ }_{a} \bar{s}^{b^{\prime}}{ }_{a^{\prime}}$, with suitable functions $s^{a}{ }_{b}$ which take values in $\operatorname{SL}(2, \mathbb{C})$.

Under a rescaling

$$
g_{\mu \nu} \rightarrow \theta^{2} g_{\mu \nu}=g_{\mu \nu}^{*}
$$

of the metric field by a positive function $\theta$ on $M$, the function $\Lambda=\Lambda(g)$ is transformed into the function $\Lambda^{*}=\Lambda\left(\theta^{2} g\right)$ according to

$$
\nabla_{\mu} \nabla^{\mu} \theta=4\left(\theta \Lambda-\theta^{3} \Lambda^{*}\right)
$$

where $\nabla_{\mu}$ denotes the torsion free covariant derivative determined by $g$. If $\Lambda^{*}$ is now an arbitrarily given function on $M$, then (7.9) provides a wave equation for $\theta$, which for suitable data on some initial surface may be solved locally to yield a positive function $\theta$ with $\Lambda\left(\theta^{2} g\right)=\Lambda^{*}$. If $\Lambda^{*}$ is to be given on $\tilde{M}$ it must be made sure that it will define a smooth function on $M$. In particular at $\{r=0\}$ the function $\Lambda^{*}$ must 
allow an expansion as described in (5.19). The simplest way to avoid problems arising from questions of smoothness is to assume that the conformal factor has been chosen such that the Ricci scalar is constant near $I_{i^{-}}$,

$$
\Lambda=\Lambda_{0}=\text { const. }
$$

The construction of the submanifold $\tilde{M}^{\prime}$ of the bundle $S(M)$ of all spin frames which are normalized with respect to the antisymmetric form $\varepsilon^{*}=\theta \varepsilon$, may be started at the spin frame $\delta^{*}{ }_{a}=\theta_{0}{ }^{-1 / 2} \delta_{a}$, where $\theta_{0}=\theta\left(i^{-}\right)$. With this choice a simple transformation law $t^{*}=t^{*}(t)$ is obtained. One finds

$$
\left.\begin{array}{rl}
\delta_{a}^{*}\left(0, r^{*}, t^{*}\right) & =\delta_{a}(0, r, t) s_{b}^{a}(r) \quad \text { with } t^{*}=t \text { and } \\
s^{a}{ }_{b}(r) & =\left(\begin{array}{cc}
\theta_{0}^{1 / 2} \theta^{-1} & \phi \\
0 & \theta_{0}^{-1 / 2}
\end{array}\right),
\end{array}\right\}
$$

where $\phi$ is some function which will not be needed in the following and the entries of the matrix have to be evaluated at $\tilde{\pi}\left(\delta_{a}(0, r, t)\right)$. This relation implies

$$
c_{00^{\prime}}^{*}\left(0, r^{*}, t\right)=\theta_{0} \theta^{-2} c_{00^{\prime}}(0, r, t), \quad r^{*}=\theta_{0}^{-1} \int_{0}^{r} \theta^{2} d r .
$$

The initial data for the field equations transform under (7.8), (7.11), (7.12) according to

$$
\begin{aligned}
\varphi_{000}^{*}\left(0, r^{*}, t\right) & =\theta_{0}^{2} \theta^{-3} \varphi_{0000}(0, r, t), \\
\phi_{000^{\prime} 0^{\prime}}^{*} & =\theta_{0}{ }^{2} \theta^{-6}\left(\theta^{2} \phi_{000^{\prime} 0^{\prime}}+2\left(H_{00^{\prime}}, \theta\right)^{2}-\theta H_{00^{\prime}}^{2} \theta\right) .
\end{aligned}
$$

The second of these equations is derived from the transformation law under (7.8)

$$
s_{\mu \nu}^{*}=s_{\mu \nu}+2 \theta^{-2}\left(\theta_{, \mu} \theta_{, \nu}-\frac{1}{4} g_{\mu \nu} \theta_{, \lambda} \theta_{, \rho} g^{\lambda \rho}\right)-\theta^{-1}\left(\theta_{; \mu \nu}-\frac{1}{4} g_{\mu \nu} \theta_{; \lambda \rho} g^{\lambda \rho}\right),
$$

where $s_{\mu \nu}=\frac{1}{2}\left(R_{\mu \nu}-\frac{1}{4} R g_{\mu \nu}\right)$ and $R_{\mu \nu}$ is the Ricci-tensor of $g_{\mu \nu}$. Finally the transformation of the value of the function $s$ at the point $i^{-}$under (7.8) is needed. Since by (1.4) the function $\Omega$ as well as its differential vanish at $i^{-}$, one finds

$$
s^{*}\left(i^{-}\right)=\theta_{0}^{-1} s\left(i^{-}\right) .
$$

If Eq. (7.9) is to be solved near $I_{1}$ - to determine the function $\theta$ for given $\Lambda^{*}$, one is free to prescribe the function $\theta$ on $I_{i^{-}}$. The relation (7.15) may be used to fix $\theta$ at $i^{-}$by assigning some appropriate value to the function $s$ at $i^{-}$. This number must be positive because of (1.7). Reading the second of Eqs. (7.13) as an ordinary differential equation for $\theta$ along the integral curves of $H_{00^{\prime}}$, one finds that it may be used to fix $\theta$ on $\tilde{N}_{0}$ near $\{r=0\}$ by prescribing a suitable value for $\phi_{000^{\prime} 0^{\prime}}$ there. This leaves open the choice of $H_{00^{\prime}} \theta$ at $\{r=0\}$ or, in the notation of (7.14), the value of $\theta_{, \mu}$ at $i^{-}$. In the case of the regular initial value problem with data being given on a null cone with respect to a space-time point $p$, this freedom may be used to give $\Sigma_{a a^{\prime}}$ any preassigned value. Since, however, by (1.4) one has $\Sigma_{a a^{\prime}}=0$ at $\{r=0\}$, there is no natural way to fix $H_{00^{\prime}} \theta$ in the present case by a local condition. This leaves the freedom of a rescaling on $I_{i}$ - by a function $\theta$ which is obtained by solving on $\tilde{N}_{0}$ the initial value problem (where (7.12) has to be taken into account),

$$
\theta^{6} \phi_{000^{\prime} 0^{\prime}}=\theta^{2} \phi_{000^{\prime} 0^{\prime}}+2\left(H_{00^{\prime}} \theta\right)^{2}-\theta H_{00^{\prime}}{ }^{2} \theta,
$$


with

$$
\theta(r=0)=1, H_{00^{\prime}} \theta(r=0)=\alpha+\sum_{k=0}^{2} \alpha_{k} T_{2}{ }^{k}{ }_{1}
$$

where the numbers $\alpha, \alpha_{k}$ are only subject to the reality conditions $\alpha=\bar{\alpha}, \alpha_{k}=(-1)^{k}$ $\bar{\alpha}_{2-k}$ but otherwise arbitrary. In addition to this 4-parameter freedom one has the 6parameter freedom to perform transformations of the type (7.1), which have to be followed up by a transformation (7.8) with (7.16) to restore the given value of $\phi_{000^{\prime} 0^{\prime}}$ on $\tilde{N}_{0}$.

\section{Determining the Constrained Data from the Free Data}

The set of functions which may be prescribed freely in an initial value problem for the conformal vacuum field equations with initial data on $\tilde{N}_{0}$ consists of

(i) the "gauge dependent data" $\Lambda, s, \phi_{000^{\prime} 0^{\prime}}$. It will be assumed here that they take the values

$$
\begin{aligned}
\Lambda & =\frac{1}{2} \text { in a neighbourhood of } \tilde{N}_{0}, \\
s & =\sqrt{2} \text { at }\{r=0, u=0\}, \\
\phi_{000^{\prime} 0^{\prime}} & =1 \text { on } N_{0} .
\end{aligned}
$$

This choice is motivated by the fact that for vanishing free data (8.2) the solution of the initial value problem in the gauge (8.1) yields the Minkowski space in the conformally rescaled form (1.1), (1.2), (1.3). Independent of the chosen free data the convergence of the null geodesics on $I_{i}$ - will be given in the gauge (8.1) by

$$
\rho=-\left\langle\omega^{1}{ }_{0}, e_{10^{\prime}}\right\rangle=-\operatorname{ctg} r .
$$

If one wants the completeness condition of Definition (1.2), (iv) to be satisfied, the free data have to be given such that they are smooth for values of $r$ in the range $0 \leqq r<\pi$.

(ii) the "free data," represented by the complex-valued function of spin-weight 2

$$
\varphi_{0}=\varphi_{0000}(0, r, t) \text { on } \tilde{N}_{0} .
$$

This function has to be given as a smooth function of $r, t$ for $0 \leqq r<\pi$, $t \in \mathrm{SU}(2)$ and such that it allows an expansion of the type (5.19), which in the present case takes the form,

$$
\left.\begin{array}{rl}
\varphi_{0} & =\sum_{p=0}^{N} \varphi_{0, p} r^{p}+0\left(r^{N+1}\right) \quad \text { for } N \in \mathbb{N}, \text { with } \\
\varphi_{0, p} & =\sum_{n=0}^{p} \sum_{i=0}^{2(n+2)} \varphi_{0, p, n, i} T_{2(n+2) n}, \quad \varphi_{0, p, n, i} \in \mathbb{C} .
\end{array}\right\}
$$

The proof of the following proposition will show how from the data (8.1), (8.2) can be determined by integration of ordinary differential equations the constrained data on $\tilde{N}_{0}$ and a formal-expansion-type solution of the conformal vacuum field equations on $\tilde{M}$. 
Proposition (8.1). Suppose that the "unknown"

$$
U=\left(b_{a a^{\prime}}, r_{a a^{\prime}}, \Gamma_{a a^{\prime} b c^{\prime}}, \varphi_{a b c d}, \phi_{a b a^{\prime} b^{\prime}}, \Omega, \Sigma_{a a^{\prime}}, s\right),
$$

the manifold $\tilde{M}$, the functions $u, r, t$ on $\tilde{M}$ etc. have been constructed from a metric $g$ and a function $\Omega$ satisfying (1.4) on a manifold $M$ with cone-like boundary $I_{-}-$as described before. Assume that the gauge dependent data take the values (8.1) and that the conformal vacuum field equations $\operatorname{Ric}\left(\Omega^{-2} g\right)=0$ are satisfied on $M \backslash I_{i}-$ near $I_{i}{ }^{-}$. If the free data $\varphi_{0}$ are known on $\tilde{N}_{0}$, then:

(i) since the gauge and the regularity conditions are satisfied, on $\{r=0, u=0\}$ the unknown $U$ can be uniquely determined from $\varphi_{0}$,

(ii) on $\tilde{N}_{0}$ the unknown $U=U_{0}$ can be determined uniquely from $\varphi_{0}$ by solving systems of (singular) ordinary differential equations along the lines $\{t=\mathrm{const}\}$. From the structure of the equations and from the fact that $\varphi_{0}$ allows the expansion (8.3), it follows that all quantities given by $U_{0}$ are smooth in the usual sense and allow expansions of the type (5.19) characterized by the values of $s$ and d given in Table 1.

(iii) in a similar way all the derivatives $\partial_{u}^{k} U=\left\{\partial_{u}^{k} b_{a a^{\prime}}, \ldots, \partial_{u}^{k} s\right\}, k \in \mathbb{N}$, of the unknown $U$ are uniquely determined on $\tilde{N}_{0}$ by the gauge and regularity conditions at $\{r=0\}$ and by the field Eqs. (6.4)-(6.11).

To obtain $U$ on $\tilde{N}_{0}$ one has to integrate Eqs. (6.4)-(6.7). Their most important feature in the present context is the singularity at $\{r=0\}$. The smoothness requirement for the tensor fields and (6.5), (6.6) imply

$$
\left.\begin{array}{c}
X_{-}\left(\varphi_{a b c 0}\right)+3 \varepsilon_{(a}{ }^{0} \varphi_{b c) 01}+\varphi_{a b c 1}=0, \\
X_{+}\left(\phi_{b c d^{\prime} 0^{\prime}}\right)+\varepsilon_{d^{\prime}} 0^{\prime} \phi_{b c 1^{\prime} 0^{\prime}}+\phi_{b c d^{\prime} 1^{\prime}}-2 \varepsilon_{(b}{ }^{1} \phi_{c) 0 d^{\prime} 0^{\prime}}=0 \text { at }\{r=0\} .
\end{array}\right\}
$$

These equations are part of Eqs. (5.12) at $\{r=0\}$. They allow one to determine $\varphi_{a b c d}$, $\phi_{a b a^{\prime} b^{\prime}}$ at $\{r=0\}$ from $\varphi_{0000}, \phi_{000^{\prime} 0^{\prime}}$. Thus by (8.4), (4.15), (4.18), (1.4), (8.1) the unknown $U$ may be obtained on $\{r=0, u=0\}$ if $\varphi_{0000}$ is known there.

To discuss Eqs. (6.4)-(6.7) on $\tilde{N}_{0}$ it is convenient to group them together and to write them as equations for the unknowns:

$$
\begin{aligned}
x_{1}= & \left(\Omega, \Sigma_{00^{\prime}}, \Gamma_{10^{\prime} 00}, \Gamma_{01^{\prime} 00}, c_{01^{\prime}}, c_{10^{\prime}},\right. \\
& \text { complex conjugates of these functions }), \\
x_{2}= & \left(\Sigma_{01^{\prime}}, \Gamma_{10^{\prime} 01}, \Gamma_{01^{\prime} 01^{\prime}} \Gamma_{11^{\prime} 00}, c_{11^{\prime}}, r_{01^{\prime}}, \Psi_{000^{\prime} 1^{\prime}}=\phi_{000^{\prime} 1^{\prime}}\right. \\
& \left.+\frac{1}{2} X_{+}\left(\phi_{000^{\prime} 0^{\prime}}\right), \psi_{0001}=\varphi_{0001^{\prime}}+\frac{1}{4} X_{-}\left(\varphi_{0000}\right), \text { c.c. }\right), \\
x_{3}= & \left(\Sigma_{11^{\prime}}, s, \gamma_{10^{\prime}}, \gamma_{01^{\prime}}, \Gamma_{11^{\prime} 01}, r_{11^{\prime}}, \Psi_{001^{\prime} 1^{\prime}}\right. \\
= & \phi_{001^{\prime} 1^{\prime}}+X_{+}\left(\phi_{000^{\prime} 1^{\prime}}\right), \Psi_{010^{\prime} 1^{\prime}}=\phi_{010^{\prime} 1^{\prime}}-\frac{1}{2} \phi_{000^{\prime} 0^{\prime}} \\
& \left.+\frac{1}{2} X_{+}\left(\phi_{010^{\prime} 0^{\prime}}\right), \psi_{0011}=\varphi_{0011}+\frac{1}{3} X_{-}\left(\varphi_{0001}\right), \text { c.c. }\right), \\
x_{4}= & \left(\gamma_{11^{\prime}}, \Psi_{011^{\prime} 1}=\phi_{011^{\prime} 1^{\prime}}-\phi_{000^{\prime} 1^{\prime}}-X_{+}\left(\phi_{010^{\prime} 1^{\prime}}\right),\right. \\
& \left.\cdot \psi_{0111^{\prime}}=\varphi_{0111}+\frac{1}{2} X_{-}\left(\varphi_{0011}\right), \text { c.c. }\right), \\
x_{5}= & \left(\Psi_{111^{\prime} 1^{\prime}}=\phi_{111^{\prime} 1^{\prime}}-2 \phi_{010^{\prime} 1^{\prime}}+X_{+}\left(\phi_{110^{\prime} 1^{\prime}}\right),\right. \\
& \left.\cdot \psi_{1111}=\varphi_{1111}+X_{-}\left(\varphi_{0111^{1}}\right), \text { c.c. }\right)
\end{aligned}
$$


Assuming that $\varphi_{0}$ is given on $\tilde{N}_{0},(8.1)$ holds, and the $x_{A}$ have been determined for $1 \leqq A<B, B \in\{1, \ldots, 5\}$, then for $x_{B}=\left(x^{1}, \ldots, x^{p}\right)$ is obtained from (6.4)-(6.7) a system of ordinary differential equations of the following type:

$$
\partial_{r} x^{i}+\frac{n_{i}}{r} x^{i}=f^{i}\left(x^{j}, r\right), \quad i=1, \ldots, p
$$

Here no summation is intended in the second term on the left. The functions $f^{i}$ are polynomials in the $x^{j}$ of order at most 2 with smooth $r$-dependent coefficients which are determined from the $x_{A}, A<B$. The crucial property of Eqs. (8.5) is that the $n_{i}$ are non-negative integers, $0 \leqq n_{i} \leqq 4$. This allows one to prove

Lemma (8.2). There exists unique smooth functions $x^{i}(r), i=1, \ldots, p$, defined for $r \geqq 0$, which satisfy (8.5) for $r>0$ and vanish at $r=0$.

The solution of (8.5) whose existence is asserted in the Lemma may be obtained by solving an integral equation. This is derived in the following way: Considering the right-hand side of (8.5) for a moment as a known continuous function of $r$, the solution of (8.5) for $r>0$ may be obtained by the standard solution formula for linear first order differential equations. The arbitrary constants in this formula are determined uniquely if $x^{i}(r)$ is required to satisfy $\lim _{r \rightarrow+0} x^{i}(r)=0$. The resulting formula for $r>0$ is

$$
x^{i}(r)=r^{-n_{i}} \int_{0}^{r}\left(r^{\prime}\right)^{n_{i}} f^{i}\left(x^{j}, r^{\prime}\right) d r^{\prime}
$$

Finding solutions of (8.5) with $\lim _{r \rightarrow+0} x^{i}(r)=0$ is thus seen to be equivalent to finding solutions of $(8.6)$, which are continuous for $r \geqq 0$. The latter problem may be solved by an iteration procedure.

For $x \in \mathbb{R}^{p}$ set $|x|=\sum_{j=1}^{p}\left|x^{j}\right|$. For chosen positive numbers $a, r_{0}$ there exists a Lipschitz constant $L>0$ such that

$$
\left|f^{i}(x, r)-f^{i}(z, r)\right| \leqq L|x-z| \forall r: 0 \leqq r \leqq r_{0}, \quad \forall x, z:|x|,|z| \leqq a .
$$

An operator $T$ which maps the functions $x^{i}(r)$ which are continuous on $\left[0, r_{0}\right]$ onto functions $(T x)^{i}(r)$ which are continuous on $\left[0, r_{0}\right]$ and vanish at $r=0$ is defined by

$$
\begin{aligned}
& (T x)^{i}(r)=r^{-n_{i}} \int_{0}^{r}\left(r^{\prime}\right)^{n_{i}} f^{i}\left(x^{j}, r^{\prime}\right) d r^{\prime} \quad \text { for } 0<r \leqq r_{0}, \\
& (T x)^{i}(0)=0 .
\end{aligned}
$$

Let $r_{1}>0$ be such that $r_{1} \leqq \min \left(r_{0}, 1 / 2 L, a / K\right)$, where $K$ is the maximum of $|f(x, r)|$ on the set $0 \leqq r \leqq r_{0},|x| \leqq a$, and define a Banach norm on the set $\left(C\left[0, r_{1}\right]\right)^{p}$ of continuous functions $x(r)=\left(x^{i}(r)\right)$ on $\left[0, r_{1}\right]$ by setting $\|x\|=\max _{0 \leqq r \leqq r_{1}}|x(r)|$. Since

$$
\left|(T x)^{i}(r)\right| \leqq \int_{0}^{r}\left|f^{i}\left(x^{j}, r^{\prime}\right)\right| d r^{\prime}
$$


the choice of $r_{1}$ implies that $T$ maps the closed ball at the origin of $\left(C\left[0, r_{1}\right]\right)^{p}$ with radius $a$ into itself and that $T$ is contractive. From this ensures the existence of a unique solution $x(r) \in\left(C\left[0, r_{1}\right]\right)^{p}$ with $T x=x$; i.e. of unique continuous functions $x^{i}(r)$ on $\left[0, r_{1}\right]$ which satisfy (8.6) for $r>0$ and vanish at $r=0$. It is clear that these functions are smooth for $r>0$. Using (8.6) one finds for $\lim _{r \rightarrow+0}\left(x^{i}(r) / r\right)$ the value $\left(n_{i}+1\right)^{-1} f^{i}(0,0)$ which coincides with the value $\lim _{r \rightarrow+0} \partial_{r} x^{i}(r)$ obtained from $(8.5)$ using (8.6). Thus $x^{j} \in C^{1}\left(\left[0, r_{1}\right]\right)$. Assuming an expansion

$$
x^{j}(r)=\sum_{k=1}^{N} a_{k}^{j} r^{k}+R_{N}^{j}(r)
$$

for $N \in \mathbb{N}$, with $a^{j}{ }_{k} \in \mathbb{C}, R_{N}{ }^{j}(r)=O\left(r^{N+1}\right)$, one finds from (8.5) that the coefficients $a^{j}{ }_{k}$ are uniquely defined and that $R^{j}{ }_{N}$ satisfies an integral equation of the type (8.6). This allows one to show that $R^{j}{ }_{N}(r)$ is $N$-times continuously differentiable. The unknown $U$ is thus obtained and smooth for $0 \leqq r<\pi, t \in \mathrm{SU}(2)$.

A straightforward though detailed inspection of the Taylor coefficients $a^{j}{ }_{k}$ in (8.7), which involves the Clebsch-Gordon expansion of the products of the functions $T_{m}{ }_{k}$, shows that the property (8.3) of $\varphi_{0}$ implies the correct expansion type of all functions. This will be discussed in more detail in [8]. The values of $d$ given in Table 1 for the frame and connection coefficients have been determined this way.

A discussion of Eqs. (5.12), (5.13) shows that the coefficients

$$
\begin{aligned}
& X_{+}\left(\varphi_{0001}\right)-\varphi_{0000}, \\
& X_{-}\left(\phi_{000^{\prime} 0^{\prime}}\right)+2 \phi_{010^{\prime} 1^{\prime}}-\phi_{000^{\prime} 0^{\prime}},
\end{aligned}
$$

of $1 / r$ in Eqs. (6.9), (6.10) must vanish at $\{r=0, u=0\}$ since the $\varphi_{a b c d}, \phi_{a b a^{\prime} b^{\prime}}$ have been determined there from (8.4). Thus Eqs. (6.9), (6.10) may be used to calculate $\partial_{u} \varphi_{0000}$, $\partial_{u} \phi_{000^{\prime} 0^{\prime}}$ as smooth functions on $\tilde{N}_{0}$ while $\partial_{u} \Omega, \partial_{u} \Sigma_{a a^{\prime}}, \partial_{u} s$ are obtained on $\{r=0\}$ from (6.11). Taking now the formal derivative of (6.4)-(6.7) by $\partial_{u}$, one finds that $\partial_{u} U$ can be found on $\widetilde{N}_{0}$ by integrating again equations of the type (8.5). Repeating this process $\partial_{u}{ }^{k} U$ is obtained for all $k \in \mathbb{N}$. Since the equation obtained by formal differentiation of (6.4)-(6.7) lead to linear systems of ordinary differential equations, the quantities $\partial_{u}^{k} U$ will be smooth for all values of $r, 0 \leqq r<\pi$.

\section{The Pure Radiation Problem as an Initial Value Problem for a Symmetric Hyperbolic System}

To formulate a regular initial value problem the field equations have to be expressed with respect to a smooth coordinate system $x^{\mu}$ and a smooth local section $M \ni x \mapsto\left(\iota_{a}(x)\right)_{a=0,1} \in S(M)$, defined in a neighbourhood of $I_{1^{-}}$. Let $\tilde{e}_{a a^{\prime}}$ be the null tetrad associated with $l_{a} \bar{l}_{a^{\prime}}, \tilde{e}^{\mu}{ }_{a a^{\prime}}=\tilde{e}_{a a^{\prime}}\left(x^{\mu}\right)$ the frame coefficients, and $\tilde{\omega}^{a}{ }_{b}$ the pullback of the connection form on $S(M)$ by that local section. Define the connection coefficients by

$$
\tilde{\Gamma}_{a a^{\prime}{ }_{c}{ }_{c}}=\left\langle\tilde{\omega}_{c}^{b}, \tilde{e}_{a a^{\prime}}\right\rangle,
$$


and introduce the notation

$$
\tilde{\gamma}_{c d}^{a b}=\frac{1}{2} \widetilde{\Gamma}_{(c}^{e^{\prime} f(a} \widetilde{\Gamma}_{d) e^{\prime}}^{b)}{ }_{f} ; \quad \tilde{\gamma}_{c^{\prime} d^{\prime}}^{a b}=\frac{1}{2} \tilde{\Gamma}_{\left({ }^{\prime}\right.}^{e}{ }^{f(a} \tilde{\Gamma}_{\left.d^{\prime}\right) e}{ }^{b)}{ }_{f} .
$$

Denote by $\tilde{\nabla}_{a a^{\prime}}$ the covariant derivative in the direction $\tilde{e}_{a a^{\prime}}$ and let $\tilde{\nabla}_{a a^{\prime}} \tilde{\Gamma}_{b b^{\prime} c d}$ be the expression which one would obtain if $\widetilde{\Gamma}_{b b^{\prime} c d}$ were the components of a spinor field. It has been shown in [7] that the conformal vacuum field equations in the gauge $\bar{\Lambda}=\frac{1}{2}$ imply for the unknown

$$
\tilde{U}=\left(\tilde{e}_{a a^{\prime}}^{\mu}, \tilde{\Gamma}_{a a^{\prime} b c}, \tilde{\varphi}_{a b c d}, \tilde{\phi}_{a b a^{\prime} b^{\prime}}, \widetilde{\Omega}, \tilde{\Sigma}_{a a^{\prime}}, \tilde{s}\right)
$$

the following system of "reduced conformal vacuum field equations" (where for lucidity the tilde is dropped now):

$$
\left.\begin{array}{c}
2 \nabla_{00^{\prime}} e_{11^{\prime}}^{\mu}-\nabla_{01^{\prime}} e^{\mu}{ }_{10^{\prime}}-\nabla_{10^{\prime}} e_{01^{\prime}}^{\mu}-2 F^{\mu}=0, \\
\left(\nabla_{00^{\prime}}+\nabla_{11^{\prime}}\right) e^{\mu}{ }_{10^{\prime}}-\nabla_{10^{\prime}} e^{\mu}{ }_{11^{\prime}}-\nabla_{10^{\prime}} e_{00^{\prime}}^{\mu}=0, \\
\left(\nabla_{00^{\prime}}+\nabla_{11^{\prime}}\right) e^{\mu}{ }_{01^{\prime}}-\nabla_{01^{\prime}}, e_{00^{\prime}}^{\mu}-\nabla_{01^{\prime}}, e_{11^{\prime}}^{\mu}=0, \\
2 \nabla_{11^{\prime}} e^{\mu}{ }_{00^{\prime}}-\nabla_{10^{\prime}} e^{\mu}{ }_{01^{\prime}}-\nabla_{01^{\prime}} e^{\mu}{ }_{10^{\prime}}-2 F^{\mu}=0,
\end{array}\right\}
$$

The system is symmetric hyperbolic if in the expression for $\nabla_{a a^{\prime}}$ the coefficients $e_{a a^{\prime}}^{\mu}$ are replaced by $\frac{1}{2}\left(e^{\mu}{ }_{a a^{\prime}}+\bar{e}^{\mu}{ }_{a a^{\prime}}\right)$. The function $F^{\mu}, F_{b}^{a}$ are given by

$$
\nabla_{v} \nabla^{v} x^{\mu}=2 F^{\mu}, \quad \delta \tilde{\omega}_{b}^{a}=-F_{b}^{a},
$$

where $\delta$ denotes the codifferential. These functions may be prescribed arbitrarily in 
an initial value problem for Eqs. (9.1)-(9.5). Together with the initial conditions they fix the coordinate system and the frame uniquely.

In the following the choice of coordinate system and frame field will be specified and the unknown $\widetilde{U}$ with respect to the new gauge will be calculated on $I_{1}-$.

Let $\delta_{a}=\delta_{a}(0,0,1)$ be the spin frame at $i^{-}$which was chosen as the starting point for the construction of $\tilde{M}$ and $c_{a a^{\prime}}=c_{a a^{\prime}}(0,0,1)$ the corresponding null tetrad. Moreover, let $x^{\mu^{\prime}}$ be the normal coordinate system centered at $i^{-}$such that $c^{\mu^{\prime}}{ }_{k}=$ $c_{a a^{\prime}}\left(x^{\mu^{\prime}}\right) \sigma^{a a^{\prime}}{ }_{k}=\delta^{\mu^{\prime}}{ }_{k}=\delta^{\mu^{\prime}}{ }_{k}$ at $i^{-}$. The coordinate system $x^{\mu}$ is fixed near $I_{l^{-}}$by the following conditions

$$
\left.\begin{array}{l}
x^{\mu}=x^{\mu^{\prime}} \text { on } I_{i^{-}} \\
\nabla_{v} \nabla^{v} x^{\mu}=2 F^{\mu}=0 \text { near } I_{i^{-}} .
\end{array}\right\}
$$

The new spin frame field $l_{a}(x)$ is required to satisfy

$$
\begin{aligned}
& l_{a}=\delta_{a} \text { at } i^{-} \\
& l_{a} \text { is parallely propagated along the null geodesics on } I_{i^{-}}, \\
& \delta \tilde{\omega}^{a}{ }_{b}=-F^{a}{ }_{b}=0 \text { near } I_{l^{-}} \text {. }
\end{aligned}
$$

The pull-back of the functions $x^{\mu}$ on $M$ by $\tilde{\pi}$ defines smooth functions

$$
x^{\mu}(u, r, t)=x^{\mu}(\tilde{\pi}(u, r, t))
$$

near $\tilde{N}_{0}$. The spin frame $l_{a}$ is described near $\tilde{N}_{0}$ by a smooth map

$$
\tilde{M} \in(u, r, t) \rightarrow s^{a}{ }_{b}(u, r, t) \in \operatorname{SL}(2, \mathbb{C}),
$$

such that

$$
l_{b}(\tilde{\pi}(u, r, t))=\delta_{a}(u, r, t) s_{b}^{a}(u, r, t)
$$

From (9.6), (9.7) it follows on $\tilde{N}_{0}$

$$
\begin{aligned}
& x^{\mu}(0, r, t)=r \delta_{k}^{\mu} \sigma_{a a^{\prime}}^{k} t_{0}^{a} \bar{t}_{0^{\prime}}^{a^{\prime}} . \\
& s_{b}^{a}(0, r, t)=t^{-1 a}{ }_{b} .
\end{aligned}
$$

This implies the relation on $\tilde{N}_{0}$

$$
\tilde{\phi}_{a b \ldots a^{\prime} b^{\prime} \ldots}\left(x^{\mu}(0, r, t)\right)=\phi_{c d \ldots c^{\prime} d^{\prime} \ldots}(0, r, t) t^{-1 c} t^{-1 d}{ }_{b \ldots} \bar{t}^{-1 c^{\prime}}{ }_{a^{\prime}} \bar{t}^{-1 d^{\prime}}{ }_{b^{\prime}}
$$

between the expression $\tilde{\phi}_{a b \ldots a^{\prime} b^{\prime} \ldots}$ of a spinor field in the new spin frame $l_{a}$ and the restriction $\phi_{a b \ldots a^{\prime} b^{\prime} \ldots}(u, r, t)$ of the corresponding spinor valued function on $S(M)$ to $\tilde{M}$. Thus the functions $\tilde{\varphi}_{a b c d}, \tilde{\phi}_{a b a^{\prime} b^{\prime}}, \widetilde{\Omega}, \bar{\Sigma}_{a a^{\prime}}, \tilde{s}$ can be calculated on $I_{i}$ - immediately from $U_{0}$ on $\tilde{N}_{0}$.

For fixed $\mu$ the differential $d x^{\mu}$ defines a spinor-valued function $e_{a a^{\prime}}^{\mu}$ on $S(M)$. On the section defined by $l_{a}$ it is given by $\tilde{e}^{\mu}{ }_{a a^{\prime}}=\tilde{e}_{a a^{\prime}}\left(x^{\mu}\right)$. Its restriction to $\tilde{M}$ is given by the function $e_{a a^{\prime}}(u, r, t)$, which can be obtained by applying the vector fields $e_{a a^{\prime}}$ on $\tilde{M}$ to the function $x^{\mu}(u, r, t)$. By (9.9) one has

$$
\tilde{e}_{a a^{\prime}}^{\mu}\left(x^{\nu}(0, r, t)\right)=t^{-1 b}{ }_{a} \bar{t}^{-1 b^{\prime}}{ }_{a^{\prime}} e_{b b^{\prime}}^{\mu}(0, r, t) .
$$

To determine the functions $e_{b b^{\prime}}^{\mu}(0, r, t)$ on $\tilde{N}_{0}$, the equation $\nabla_{v} \nabla^{v} x^{\mu}=0$ may be 
expressed in terms of these functions and the vector fields $e_{a a^{\prime}}$ on $\tilde{M}$ to obtain

$$
\left\langle d e^{\mu}{ }_{b b}-\omega_{b}^{c} e_{c b^{\prime}}^{\mu}-\bar{\omega}^{c^{\prime}}{ }_{b^{\prime}} e^{\mu}{ }_{b c^{\prime}}, e_{a^{\prime}}^{b}\right\rangle=0 .
$$

From (9.9), (4.14) one has on $\tilde{N}_{0}$,

$$
e^{\mu}{ }_{00^{\prime}}(0, r, t)=\delta^{\mu}{ }_{k} \sigma_{a a^{\prime}}^{k} t^{a}{ }^{a} t^{a^{\prime}}{ }^{\prime} .
$$

With (4.14), (4.17), (4.18), Eqs. (9.11) for $a^{\prime}=0$ take the form

$$
\partial_{r} e^{\mu}{ }_{1 b^{\prime}}+\frac{1}{r}\left(X_{-}\left(e_{0 b^{\prime}}^{\mu}\right)+e^{\mu}{ }_{1 b^{\prime}}-\varepsilon_{b^{\prime}}{ }^{1^{\prime}} e^{\mu}{ }_{00^{\prime}}\right)-e_{10^{\prime}}^{*}\left(e^{\mu}{ }_{0 b^{\prime}}\right)=e_{0 c^{\prime}}^{\mu} \bar{\Gamma}_{10^{\prime}{ }^{c^{\prime}} b^{\prime}}+e^{\mu}{ }_{c b^{\prime}} \Gamma_{10^{\prime}{ }^{c}{ }_{0} .} .
$$

By (5.12) the factors of $1 / r$ must vanish at $\{r=0\}$ and allow one to determine $e^{\mu}{ }_{a a^{\prime}}$ there from (9.12). Introducing these factors as new unknowns in Eqs. (9.13) one is again led to equations of the type (8.5). Hence (9.13) has unique smooth solutions on $\widetilde{N}_{0}$ which together with (9.10) determine the functions $\tilde{e}^{\mu}{ }_{a a^{\prime}}$ on $I_{i^{-}}$.

Relation (9.8) and the transformation law for connection forms imply the equation

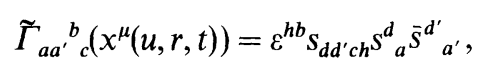

with

$$
s_{d d^{\prime} e f}=\left\langle d s^{a}{ }_{e}+\omega^{a}{ }_{c} s_{e}^{c}, e_{d d^{\prime}}\right\rangle \varepsilon_{a h^{\prime}} s^{h} .
$$

The terms in brackets is the covariant derivative of $s^{a}{ }_{b}$ in the direction of $e_{c c^{\prime}}$, if for fixed lower index $s^{a}{ }_{b}$ is considered as a spinor-valued function. Furthermore for fixed indices of the quantity $s_{c c^{\prime} \text { ef }}$ transforms on $\{r=0, u=0\}$ under SU(2) like a spinorvalued function. By (9.9) one has $s_{00^{\prime} \text { ef }}=0$ on $\widetilde{N}_{0}$, thus by (5.12)

$$
s_{a a^{\prime} e f}=0 \quad \text { at }\{r=0, u=0\} \text {. }
$$

Since the fields $e_{00^{\prime}}, e_{01^{\prime}}$ are tangent to $\tilde{N}_{0}$ and $s^{a}{ }_{b}$ is given on $\tilde{N}_{0}$ by (9.9), the new connection coefficients will be known on $I_{1}$ - from (9.14) as soon as $s_{11^{\prime} b c}$ has been determined on $N_{0}$. The equation $\delta \omega^{a}{ }_{b}=0$ can be written on $\tilde{M}$ as equation for $s_{a a^{\prime} e f}$,

$$
\left\langle d s_{c c^{\prime} e f}-\omega^{h}{ }_{c h} s_{h c^{\prime} e f}-\bar{\omega}^{h^{\prime}}{ }_{c^{\prime}} s_{c h^{\prime} e f}, e^{c c^{\prime}}\right\rangle=0 .
$$

Using that by (9.15) Eq. (9.17) involves twofold covariant differentials and that these can be expressed in terms of the original form and the curvature form, Eq. (9.17) can be written as equation for $s_{11^{\prime} a b}$ which is of the type

$$
\partial_{r} s_{11^{\prime} a b}+\frac{1}{r} s_{11^{\prime} a b}=f_{a b}\left(s_{11^{\prime} a b}, r\right) .
$$

The function $f_{a b}$ is a smooth function determined from $s^{a}{ }_{b}, s_{a a^{\prime} b c}$ with $a a^{\prime} \neq 11^{\prime}$, known directional derivatives of these functions and from $U$ on $\widetilde{N}_{0}$. By Lemma (8.2) Eq. (9.18) has a unique smooth solution $s_{11^{\prime} \text { ef }}$ satisfying (9.16).

Collecting results one arrives at

Proposition (9.1). Let free data $\varphi_{0}(0, r, t)$ satisfying (8.3) be given arbitrarily but smooth for $0 \leqq r<\pi, t \in \mathrm{SU}(2)$, and assume that the gauge dependent data satisfy (8.1). 
Then the initial data set

$$
\tilde{U}_{0}=\left(\tilde{e}_{a a^{\prime}}, \tilde{\Gamma}_{a a^{\prime} c}^{b}, \tilde{\varphi}_{a b c d}, \tilde{\phi}_{a b a^{\prime} b^{\prime}}, \widetilde{\Omega}, \tilde{\Sigma}_{a a^{\prime}}, \tilde{s}\right)
$$

for the reduced conformal vacuum field Eqs. (9.1)-(9.5) in the gauge (9.6), (9.7) can be determined uniquely as smooth functions on $I_{1}^{-}$, such that

$$
\begin{aligned}
\varphi_{0}(0, r, t) & =\tilde{\varphi}_{a b c d}\left(x^{\mu}(0, r, t)\right) t^{a}{ }_{0} t^{b}{ }_{0} t^{c}{ }_{0} t^{d}{ }_{0}, \\
1 & =\tilde{\phi}_{a b a^{\prime} b^{\prime}}\left(x^{\mu}(0, r, t)\right) t^{a}{ }_{0} t^{b}{ }_{0} \bar{t}^{a^{\prime}{ }^{\prime}{ }^{\prime} \bar{t}^{b^{\prime}}{ }_{0^{\prime}},}
\end{aligned}
$$

with $x^{\mu}(0, r, t)$ being given by (9.9).

The significance of the data set $\widetilde{U}_{0}$ and the reduced equation follows from

Proposition (9.2). A solution $\tilde{U}$ near $I_{i}$ - of the reduced conformal vacuum field Eqs. (9.1)-(9.5) in the gauge (9.6), (9.7), which coincides on $I_{i}-$ with the value $\widetilde{U}_{0}$ referred to in Proposition (9.1), is a solution of the conformal vacuum field equations. It provides a solution of Einstein's vacuum field equations with complete null cone at past timelike infinity $i^{-}$.

The proof of this proposition follows the same pattern as in the case where data are described on two intersecting null hypersurfaces [5] or on a space-like hypersurface $[5,7]$. The only difference between those cases and the present situation is that in the latter one has occasionally to switch back and forth between the two types of gauge conditions to show that the way the data $\tilde{U}_{0}$ have been determined and the fact that $\tilde{U}$ is a solution of (9.1)-(9.5) with (9.6), (9.7) imply that the field equations are satisfied on $I_{1^{-}}$. Here again equations of the type (8.5) turn up. The subsidiary system used to show that the field equations are indeed satisfied near $I_{i^{-}}$is derived in the same way as discussed in $[5,7]$. Furthermore the local uniqueness theorem for the subsidiary equations with data on $I_{i}$ - is proved by the techniques discussed in [6]. The details will not be reproduced here. The uniqueness property of the system (9.1)-(9.5) allows one to state

Proposition (9.3). A solution of Einstein's vacuum field equations with complete null cone at past timelike infinity $i^{-}$in the gauge (8.1) is uniquely determined by the free data $\varphi_{0}(0, r, t)$, if the functions $r, t$ and the frame with respect to which $\varphi_{0}, \phi_{00}$ are expressed have the meaning discussed before.

By Propositions (9.1)-(9.3) the pure radiation problem has been reduced to the task of showing the existence of a solution of the reduced Eqs. (9.1)-(9.5) with (9.6), (9.7) for the initial data $\widetilde{U}_{0}$ provided in Proposition (9.1).

Acknowledgements. I should like to thank the members of the relativity groups in Hamburg and Munich for discussions.

\section{References}

1. Bondi, H., van der Burg, M. G. J., Metzner, A. W. K.: Gravitational waves in general relativity. VII Waves from axi-symmetric isolated systems. Proc. Roy. Soc. Lond. A 269, 21-52 (1962)

2. Bruhat, Y.: Problème des conditions initiales sur un conoide charactéristique. C.R. Acad. Sci. 39713973 (1963)

3. Friedlander, F. G.: The wave-equation on a curved space-time. Cambridge: Cambridge University Press 1975 
4. Friedrich, H.: Eine Untersuchung der Einsteinschen Vakuumfeldgleichungen in der Umgebung regulärer und singulärer Nullhyperflächen. Thesis, University of Hamburg (1979)

5. Friedrich, H.: The asymptotic characteristic initial value problem for Einstein's vacuum field equations as an initial value problem for a first order quasilinear symmetric hyperbolic system. Proc. Roy. Soc. Lond. A 378, 401-421 (1981)

6. Friedrich, H.: Cauchy problems for the conformal vacuum field equations in general relativity. Commun. Math. Phys. 91, 445-472 (1983)

7. Friedrich, H.: On the hyperbolicity of Einstein's and other gauge field equations. Commun. Math. Phys. 100, 525-543 (1985)

8. Friedrich, H.: The pure radiation problem for analytic data. (In preparation)

9. Friedrich, H., Stewart, J.: Characteristic initial data and wave front singularities in general relativity. Proc. Roy. Soc. Lond. A 385, 345-371 (1983)

10. Geroch, R.: Asymptotic Structure of Space-Time. In: Esposito, F. R., Written, L. (eds.). Asymptotic structure of space-time. New York: Plenum Press 1976

11. Geroch, R., Horowitz, G.T.: Asymptotically simple does not imply asymptotically minkowskian. Phys. Rev. Lett. 40, 203-206 (1978)

12. Newman, E., Penrose, R.: An approach to gravitational radiation by a method of spin coefficients. J. Math. Phys. 3, 566-578 (1962)

13. Newman, E., Penrose, R.: Note on the Bondi-Metzner-Sachs group. J. Math. Phys. 7, 863-870 (1966)

14. Penrose, R.: Null hypersurface initial data for classical fields of arbitrary spin and for general relativity, published 1963 in Aerospace Res. Lab. Tech. Doc. Rep. 63-56 (P. G. Bergmann) reprinted in G.R.G. 12, 225-264 (1980)

15. Penrose, R.: Asymptotic properties of fields and space-times. Phys. Rev. Lett. 10, 66-68 (1963)

16. Penrose, R.: Conformal treatment of infinity. In: DeWitt, DeWitt (eds.) . Relativity, groups and topology. New York: Gordon \& Breach 1964

17. Penrose, R.: Zero rest-mass fields including gravitation: Asymptotic behaviour. Proc. Roy. Soc. Lond. A 284, 159-203 (1965)

18. Penrose, R.: Structure of space-time. In: DeWitt, C. M., Wheeler, J. A. (eds.). Battelle rencontres. New York: W. A. Benjamin 1967

19. Penrose, R.: Relativistic symmetry groups. In: Barut, A. O. (ed.). Group theory in non-linear problems. Amsterdam: D. Reidel 1974

20. Pirani, F. A. E.: Invariant formulation of gravitational radiation theory. Phys. Rev. 105, 1089-1099 (1957)

21. Sachs, R. K.: Gravitational waves in general relativity. VI The outgoing radiation condition. Proc. Roy. Soc. Lond A 264, 309-338 (1961)

22. Sachs, R. K.: Gravitational waves in general relativity. VIII Waves in asymptotically flat space-time. Proc. Roy. Soc. Lond. A 270, 103-126 (1962)

Communicated by S. W. Hawking

Received June 5, 1985 
